\title{
Sur les Singularités Essentielles et Isolées des Applications Holomorphes à Valeurs dans une Surface Complexe
}

\author{
A notre Professeur Kiyoshi Oka, décédé le $1^{\text {er }}$ mars 1978 matin
}

Par

Toshio Nishino* et Masakazu SuzuKI*

\section{Table des Matières}

Introduction.

Chapitre I - Quelques propriétés générales de l'ensemble limite (cluster set) $f(0 ; M)$.

1. Définition de $f(0)=f(0 ; M)$ (Théorème 1). .................................. 463

2. Rapport avec la pseudo-distance de Kobayashi (Théorème 2). ............... 464

3. Cas où $f(0)$ est une courbe analytique (Théorème 3 ). ..........................465

Chapitre II - Cas où $f(0)$ est une courbe analytique compacte dans une surface complexe.

4. Courbes de valeurs exceptionnelles et $l(\tilde{P})$ (Théorème 4). ...................... 469

5. Ensemble limites d'une suite d'images analytiques du disque fermé $\bar{\Delta}$ :



6. Surfaces de recouvrement d'Ahlfors. ............................................. 479

7. Liste des types de $f(0)$ compacts compris dans une courbe de valeurs exceptionnelles (Théorème 5).

Chapitre III - Singularités essentielles des isomorphismes analytiques le long d'une courbe compacte.

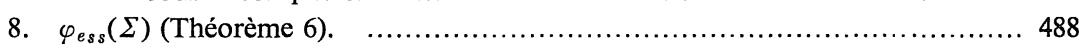

9. Rapport avec la dimension de Kodaira logarithmique (Théorème 7). ….... 491 Bibliographie.

\section{Introduction}

Soit $f$ une application holomorphe d'un disque pointé $\Delta^{*}=\{z \in \mathbb{C}|0<| z \mid$. $<1\}$ tracé sur la droite complexe $\boldsymbol{C}$ (ou le plan d'une variable complexe $z$ ) dans une variété analytique complexe $M$. Désignons par $f(0 ; M)$ l'ensemble des points $a$ de $M$ pour lesquels il existe une suite de points $z_{n}$ de $\Delta^{*}$ tendant vers

Communiqué par S. Nakano, le 25 juillet, 1978. Revu le 5 décembre, 1978.

* Faculté de Technologie, Université de Kyushu. 
$z=0$ et vérifiant $\lim _{n \rightarrow \infty} f\left(z_{n}\right)=a .^{(1)}$

Lorsque $f(0 ; M)$ contient au moins deux points, on dira que $f$ a une singularité essentielle à l'origine $z=0$.

Dans le cas où $M$ est de dimension complexe un, M. Ohtsuka [17] a montré que, si $z=0$ est un point singulier essentiel de $f$, alors tout point de $M$ appartient à $f(0 ; M)$, et de plus, $M$ doit être analytiquement isomorphe ou bien à la droite projective $\boldsymbol{P}^{1}$ (la sphère de Riemann), ou bien à $\boldsymbol{P}^{1}-\{$ un ou deux points $\}$, ou bien à un tore complexe. On peut considérer cette dernière assertion comme une généralisation du grand théorème de Picard, la première assertion correspondant à un théorème de Weierstrass. L'objet essentiel de ce travail est l'étude de ce genre de phénomène dans le cas où $M$ a deux (ou plus de deux) dimensions complexes. Nous donnerons également une application de nos résultats sur $f(0 ; M)$ à quelques questions sur les automorphismes analytiques de surfaces complexes.

Dans le premier Chapitre ( $\mathrm{n}^{\text {os }} 1$ à 3 ), nous montrerons que cet ensemble $f(0 ; M)$ a quelques propriétés remarquables même au cas où la dimension complexe de $M$ est $\geqq 2$ (cf. les Théorèmes 1 , 2, et 3 , ci-dessous). Signalons la pseudoconcavité de $f(0 ; M)$ (Théorème 1 , où $\operatorname{dim} . M=2)$, qui se résulte de la pseudoconvexité des domaines de normalité des familles d'hypersurfaces analytiques trouvée dans la théorie des fonctions de plusieurs variables complexes ([19], voir aussi [14]).

Dans le Chapitre II ( $\mathrm{n}^{\text {os }} 4$ à 7 ), nous étudierons en particulier le cas où $M$ est une surface analytique complexe non-singulière $S$ et où $C=f(0 ; S)$ est une courbe analytique compacte dans $S .^{(2)}$ Signalons l'inégalité parue dans notre Théorème $4\left(n^{\circ} 4\right)$ qui exprime une condition pour le nombre des lieux par lesquels passent des courbes analytiques de valeurs exceptionnelles (cf. la Définition 2 au $n^{\circ} 4$ ) de $f$; elle a la même forme que l'inégalité trouvée par R. Nevanlinna et L. Ahlfors pour les ramifications complètes (cf., par exemple, la formule (67) de [25]). Les numéros 5 et 6 seront consacrés à la démonstration du Théorème 4. Au numéro 7, nous considérerons les courbes analytiques compactes $C$ (n'ayant que des points doubles ordinaires comme points singuliers et minimales) dans $S$ telles qu'il y ait une application holomorphe $f$ de $\Delta^{*}$ dans $S-C$ vérifiant $f(0 ; S)=C$. L'inégalité dite plus haut nous permet de faire une

(1) "cluster set of $f$ at $z=0$ ".

(2) Un sous-ensemble analytique de $S$ de dimension complexe pure 1 sera appelé simplement courbe analytique dans $S$. 
liste contenant tous les types de ces courbes $C$ (Tableaux 1, 2 et 3 au $n^{\circ} 7$ ).

Dans le dernier Chapitre III, nous appliquerons ces résultats à quelques questions sur les automorphismes analytiques de surfaces complexes: En considérant le complément $V=S-C$ d'une courbe analytique compacte $C$ dans une surface complexe $S$, on étudiera les singularités essentielles le long de $C$ des isomorphismes analytiques de $V$ (Théorème 6 au $\mathrm{n}^{\circ} 8$ ); puis, dans le cas où $S$ est compacte, on mentionnera leur rapport avec l'invariant $\bar{\kappa}(V)$ (logarithmic Kodaira dimension) de $V$ introduit par S. Iitaka [8]. On retrouvera ici le résultat de F. Sakai [24], dans le cas de surfaces complexes: si $V$ est de type générale (c'est-à-dire $\bar{\kappa}(V)=2$ ), tout automorphisme analytique de $V$ est la restriction sur $V$ d'une transformation biméromorphe de $S$ (Corollaire au Théorème $\left.7, \mathrm{n}^{\circ} 9\right)$. Pour terminer, nous avouons que la recherche de $f(0 ; M)$ de ce travail était motivée au début par le but de l'appliquer à quelques questions concernant les automorphismes analytiques de l'espace de deux variables complexes $\mathbb{C}^{2}$, qu'étudiait T. Kizuka à cette époque (cf. [9]).

\section{Chapitre I. - Quelques Propriétés Générales de l'Ensemble Limite (cluster set) $f(0 ; M)$}

1. Définition de $f(0)=f(0 ; M)$ 。 On désignera par $\Delta_{\rho}^{*}$ le disque pointé $0<|z|<\rho$ de rayon $\rho>0$ dans le plan $\mathbb{C}$ d'une variable complexe $z$. On écrira $\Delta^{*}$ au lieu de $\Delta_{1}^{*}$. Soit $f: \Delta^{*} \rightarrow M$ une application holomorphe de $\Delta^{*}$ dans une variété analytique complexe ${ }^{(3)} M$ de dimension complexe $n$. Définissons l'ensemble limite (cluster set) $f(0 ; M)$ dans $M$ de $f(z)$ à l'origine $z=0$ par:

$$
f(0 ; M):=\bigcap_{\rho>0} \overline{f\left(\Delta_{\rho}^{*}\right)},
$$

où $\overline{f\left(\Delta_{\rho}^{*}\right)}$ est l'adhérence de $f\left(\Delta_{\rho}^{*}\right)$ dans $M$. On écrira aussi $f(0)$ au lieu de $f(0 ; M)$.

Si $f(0)$ (fermé) est compact, il est connexe.

Proposition 1. $f$ se prolonge en une application holomorphe $\bar{f}$ de $\Delta$ : $|z|<1$ dans $M$ si (et seulement si) $f(0 ; M)$ est un seul point de $M$.

En effet, la proposition est une conséquence immédiate du "Riemann's theorem on remouvable singularities".

(3) On supposera dans tout ce qui suit que les variétés sont dénombrables à l'infini. 
Le théorème suivant montre que la forme de cet ensemble $f(0)$, supposé contenant au moins deux points, est soumis à une forte restriction:

Théorème $1^{(4)}$. Soit $M$ une variété analytique complexe de dimension complexe 2. Si $f(0)$ d'une application holomorphe $f$ de $\Delta^{*}$ dans $M$ contient au moins deux points, cet ensemble $f(0)$ est pseudoconcave en chaque point de $f(0)$.

(On dit ici que $f(0)$ est pseudoconcave en un point $a$ de $f(0)$, s'il existe un voisinage $U$ de $a$ tel que $U-f(0)$ soit pseudoconvexe ${ }^{(5)}$ ou bien vide).

En effet, supposons qu'il existe un ouvert $U$ de $M$ analytiquement isomorphe à un dicylindre: $|x|<1,|y|<1$ tel que $f(0) \cap U \neq \emptyset, f(0) \cap U^{*}=\varnothing$, où

$$
\begin{aligned}
U^{*}= & \{P \in U|| x(P) \mid<r\} \cup\{P \in U|| x(P)|<1, s<| y(P) \mid<1\}, \\
& (0<r<1,0<s<1) .
\end{aligned}
$$

$X(z)=x(f(z)), Y(z)=y(f(z))$ sont des fonctions holomorphes dans $D=f^{-1}(U)$ (non vide). Prenons un nombre réel $\varepsilon>0$ tel que l'on ait $\varepsilon<r<1-\varepsilon, s<1-2 \varepsilon$ et $f(0) \cap U_{\varepsilon} \neq \emptyset$, où

$$
U_{\varepsilon}=\{P \in U|| x(P)|<1-\varepsilon,| y(P) \mid<1-\varepsilon\} .
$$

Considérons dans $\Delta_{\rho}^{*}(0<\rho<1)$ l'image réciproque $f^{-1}\left(U_{\varepsilon}\right) \cap \Delta_{\rho}^{*}=D_{\rho}$ (non vide). Puisque $f\left(\Delta_{\rho}^{*}\right) \subset U_{\varepsilon}$ entraîne $f(0)=$ un point $\}$, on peut supposer $D_{\rho} \neq \Delta_{\rho}^{*}$ pour tout $\rho(>0)$. Donc, l'origine $z=0$ appartient à l'adhérence de la frontière $\gamma$ de $D_{\rho}$ dans $\Delta_{\rho}^{*}$. Or, d'après l'hypothèse $f(0) \cap U^{*}=\varnothing$, on peut trouver un $\rho>0$ tel que l'on ait:

(i) $|X(z)|>\varepsilon$ pour $z \in D_{\rho}$

et $|Y(z)|<1-2 \varepsilon$ pour $z \in D_{\rho}$, de sorte que

(ii) $|X(\zeta)|=1-\varepsilon$ pour $\zeta \in \gamma$.

On a donc, d'après le théorème d'Iversen (cf. Theorem 1, p. 14 de [16]), $\lim _{z \rightarrow 0}|X(z)|=1-\varepsilon$; d'où $f(0) \cap U_{\varepsilon}=\emptyset$, ce qui est contraire à la définition de $\varepsilon>0$. $\underset{z \in D}{z \rightarrow 0}$

Donc, $f(0)$ est pseudoconcave.

2. Rapport avec la pseudo-distance de Kobayashi. Désignons par $d_{M}(P, Q)$ $(P, Q \in M)$ la pseudo-distance intrinsèque de $M$ introduite par S. Kobayashi

(4) Comme on l'a déjà remarqué dans l'Introduction, ce théorème se résulte également du résultat de [19] ou de [14].

(5) Pour la notion de pseudoconvexité, voir [20]. 
(cf. [10], Chap. IV). Nous dirons qu'un $P \in M$ est un point hyperbolique de $M$, si l'on a $d_{M}(P, Q)>0$ pour tout $Q \neq P$, et désignerons par $A(M)$ l'ensemble des points non-hyperboliques de $M$. Soit $U$ un polycylindre fermé: $\left|x_{i}\right| \leqq \rho$ $(i=1, \ldots, n, \rho>0)$ de centre $P \in M$ dans un voisinage de coordonnées locales $x_{i}$ de $M$. Alors, d'après H. L. Royden [22], $P$ est un point hyperbolique de $M$, si et seulement si $\inf _{Q \in \partial U} d_{M}(P, Q)>0$. Compte tenu de la continuité de $d_{M}(P, Q)$ par rapport aux $\stackrel{Q}{P}, Q$, , il en résulte que $A(M)$ esi fermé (et que $d_{M}(P, Q)$ induit sur $M-A(M)$ la topologie usuelle. Cf. [22] Theorem 2.) On a la

Proposition 2. Soit $f$ une application holomorphe de $\Delta^{*}$ dans $M$. Si $f(0)$ contient au moins deux points, on a alors $f(0) \subset A(M)$. (Voir [10], Chap VI).

Or, R. Brody [2] a montré que, si $A(M)$ est un sous-ensemble compact (non vide) de $M$, il existe une application holomorphe non-constante $g: \mathbb{C} \rightarrow M$ (evidemment, on a alors $g(\boldsymbol{C}) \subset A(M)$ ). De la même manière qu'il a procédé (que nous ne répétons pas ici), on voit que:

Théorème 2. Si $f(0)$ d'une application holomorphe $f$ de $\Delta^{*}$ dans $M$ est compact et contient au moins deux points, il contient l'image $g(\mathbb{C})$ d'une application holomorphe non-constante $g$ de $C$ dans $M$.

(Pour la démonstration, nous nous contentons de renvoyer le lecteur à [2]). En tenant compte du théorème d'Ohtsuka [17] cité dans l'Introduction, on en déduit le corollaire suivant:

Corollaire. Si $f(0)$ d'une application holomorphe $f$ de $\Delta^{*}$ dans $M$ est une courbe analytique irréductible et compacte, elle est de genre $\leqq 1$.

Ce fait-ci nous a été indiqué par T. Ueda; son idée de démonstration était d'appliquer le résultat de $\mathrm{T}$. Nishino [15] et cette indication nous a donné un point de départ pour ce travail.

3. Cas où $f(0)$ est une courbe analytique. Soit $M$ une variété analytique complexe de dimension complexe $n \geqq 2$. Un sous-ensemble analytique de $M$ de dimension (complexe) pure un sera appelé simplement courbe analytique dans M.

Il convient de citer ici un théorème dû à $P$. Thullen [28] et à R. RemmertK. Stein [21] sur les singularités essentielles des sous-ensembles analytiques. On dira qu'un sous-ensemble $E$ de $M$ est analytique en un point $P \in M$, s'il 
existe un voisinage $U=U(P)$ de $P$ tel que $E \cap U$ soit un sous-ensemble analytique de $U$ (y compris vide). On a alors, le

Théorème ([28], $[21])$. Soit $A$ un sous-ensemble analytique irréductible de $M$, et $\sigma$ un sous-ensemble analytique de $M-A$ de dimension pure. Supposons que l'adhérence $\bar{\sigma}$ de $\sigma$ dans $M$ n'est pas analytique en un point $P_{0}$ de A. Alors, on $a \operatorname{dim} A \geqq \operatorname{dim} \sigma . \quad$ De plus, si $\operatorname{dim} A=\operatorname{dim} \sigma, \bar{\sigma} n ' e s t$ analytique en aucun point de $A$ (en particulier, on $a A \subset \bar{\sigma})$.

De ce théorème, on a aussitôt:

Proposition 3. Si $f(0)$ d'une application holomorphe de $\Delta^{*}$ dans $M$, contenant au moins deux points, est compris dans une courbe analytique $C$ dans $M$, alors $f(0)$ lui-même est une courbe analytique dans $M$, de sorte que $f(0)$ se décompose en des composantes irréductibles de $C$.

En effet, si $f\left(\Delta^{*}\right) \subset C$, la proposition se résulte du théorème d'Ohtsuka cité dans l'Introduction. Supposons $f\left(\Delta^{*}\right) \not \subset C$. On peut alors trouver $0<\rho<1$ de façon que l'image $K_{\rho}$ du cercle $\partial \Delta_{\rho}:|z|=\rho$ par $f$ se trouve dans $M-C$. Soit $M_{0}=M-K_{\rho}$. D'après l'hypothèse $f(0) \subset C$, l'image $\sigma=f\left(\Delta_{\rho}^{*}\right)$ est une courbe analytique dans $M_{0}-C$. Or, comme $f(0)$ contient au moins deux points, $\bar{\sigma}$ (l'adhérence dans $M_{0}$ ) n'est pas analytique en un certain point de $C$. Donc, d'après le Théorème que nous venons de cité ci-dessus, $f(0)(=1$ 'ensemble des points de $C$ en lesquels $\bar{\sigma}$ n'est pas analytique) est composé d'un certain nombre $(\leqq \infty)$ de composantes irréductibles de $C$.

C.Q.F.D.

Considérons maintenant le cas où $f(0)$ est une courbe analytique dans $M$. Une courbe analytique $C$ dans $M$ sera dite de genre $p$, si son modèle non-singulier (la normalisée de $C$ ) est de genre $p$.

Théorème 3. Si $f(0)$ d'une application holomorphe $f$ de $\Delta^{*}$ dans $M$ coïncide avec une courbe analytique $C$ dans $M$, toute composante irréductible de $C$ est de genre zéro ou un.

Pour démontrer ce théorème, préparons un lemme:

Lemme 1. Soit $C$ une courbe analytique non-singulière n'ayant aucune composante compacte dans $M$, et $K$ un sous-ensemble compact de $C$. Il existe alors, un sous-ensemble compact $T(K)$ de $M$ et une application $\varphi$ de $T(K)$ sur $K$ vérifiant les conditions suivantes:

(i) $T(K) \cap C=K$ 
(ii) $\varphi$ est la restriction sur $T(K)$ d'une application holomorphe d'un voisinage de $T(K)$ sur un voisinage de $K$ dans $C$ telle que $\left.\varphi\right|_{K}=\mathrm{id}$.

(iii) le triple $(T(K), \varphi, K)$ est un espace fibré topologique localement trivial de base $K$ et à fibres isomorphes à un disque fermé de dimension réelle $2 n-2, n$ étant la dimension complexe de $M$.

(Nous appellerons ce $T(K)$ tube le long de $K$ et $\varphi: T(K) \rightarrow K$ sa projection ou retraction sur $K$. La réunion $\bigcup_{P \in K} \partial \varphi^{-1}(P)$ des bords des fibres de $\varphi$ sera appelée le bord de $T=T(K)$ et désignée par $b T)$.

En effet, $C$ étant une variété de Stein, il existe, d'après Y.-T. Siu [26] (ou M. Suzuki [27], Lemme 3), un ouvert $V$ de $M$ de Stein contenant $K$. Pour démontrer le Lemme 1, il suffira donc de le prouver en supposant que $M$ est une variété de Stein. Or, ceci se résulte du Hilfssatz 11 de O. Forster et K. J. Ramspott [3]. Mais, comme ce lemme joue un rôle important dans cet article, nous allons donner une démonstration simple ci-dessous (en supposant $M$ de Stein): Comme $C$ est une courbe non-singulière et n'a pas de composante compacte, il existe, d'après R. C. Gunning et R. Narasimhan [4], une fonction holomorphe $h$ sur $C$ telle que: (*) sa différentielle $d h$ ne s'annule en aucun point de $C$. Or, $M$ étant de Stein, on peut trouver, d'après la théorie des ideaux de fonctions analytiques dû à $\mathrm{K}$. Oka et à $\mathrm{H}$. Cartan, une fonction holomorphe $H$ sur $M$ telle que $H_{\mid C}=h$. En suite, compte tenue de la condition (*) ci-dessus pour $d h$, il existe un voisinage $V^{\prime}$ de $C$ telle que les variétés définies par $H=$ const. soient régulières dans $V^{\prime}$. En introduisant une métrique hermitienne sur $M$, désignons par $d(P, Q)$ la distance de deux points $P, Q \in M$, et considérons

$$
\lambda=\inf \{d(P, Q) \mid P, Q \in K, P \neq Q, H(P)=H(Q)\} .
$$

Puisque $K$ est compact et les variétés $H=$ const. coupent $C$ transversalement, on a $\lambda>0$. Soit $0<\varepsilon<\lambda / 3$, et

$$
F(P)=\left\{Q \in V^{\prime} \mid H(Q)=H(P), d(P, Q) \leqq \varepsilon\right\}
$$

pour chaque $P \in K$; alors $P \neq Q$ implique $F(P) \cap F(Q)=\emptyset$. Posons $T(K)=$ $\cup F(P)$, et désignons par $\varphi$ l'application $T(K) \rightarrow K$ qui, à chaque point $Q \in$ $T(K)$, associe le point $P \in K$ tel que $Q \in F(P)$. Il est clair que $\varphi$ se prolonge en une application holomorphe $V \rightarrow C$ d'un voisinage $V$ de $T(K)$ dans $C$ et que $T(K) \cap C=K, \varphi_{\mid K}=$ id. Quant'à la dernière condition (iii), vu $(d H)_{\mid C} \neq 0$, il suffit de prendre $\varepsilon>0$ assez petit. Et la démonstration du Lemme 1 est achevéc. 
Citons un autre résultat d'Ohtsuka [18] mot à mot: 《Soient $G$ un ouvert dans le plan $z, z_{0} \in \partial G$ et $K$ un sous-ensemble compact de $\partial G$ contenant $z_{0}$. Soit $f$ une application de $G$ dans une surface de Riemann $R$. Nous définissons le cluster set $f\left(z_{0} \mid G\right)^{(6)}$ en $z_{0}$ par

$$
\bigcap_{U \in \mathfrak{u}(z 0)} \overline{f(U \cap G)},
$$

où $\mathfrak{U}\left(z_{0}\right)$ est le système de voisinages de $z_{0}$ et $\overline{f(U \cap G)}$ est l'adhérence de $f(U \cap G)$, et le boundary cluster set $f\left(z_{0} \mid \partial G-K\right)$ par

$$
\widehat{U \in \mathfrak{U}\left(z_{0}\right)} \underset{z \in U \cap(\partial G-K)}{\bigcup} f(z \mid G) 》 .
$$

On a alors, le

《Théorème. Soit $f$ une application holomorphe d'un ouvert $G d u$ plan $z$ dans une surface de Riemann $R, z_{0} \in \partial G$ et $K$ un sous-ensemble compact de capacité logarithmique nulle qui contient $z_{0}$ et qui est contenu dans une composante connexe de $\partial G$. Si $f\left(z_{0} \mid G\right)$ contient plus d'un point, alors $f\left(z_{0} \mid G\right)$ $-f\left(z_{0} \mid \partial G-K\right)$ est ouvert, et toute composante $D$ de $f\left(z_{0} \mid G\right)-f\left(z_{0} \mid \partial G-K\right)$ est de genre $\leqq 1$. Si le genre de $D$ est 0 (resp. 1), alors $f$ prend, dans tout voisinage de $z_{0}$, tous les points de $D$ à l'exception de deux points au plus (resp. sans exceptions).》

Nous pouvons maintenant démontrer le Théorème 3: En effet, supposons que $C$ possède une composante $C_{0}$ de genre $\geqq 2$. Soit $k$ l'ensemble des points singuliers de $C$ ( $k$ est discret). Prenons un ouvert connexe, non-compact et relativement compact $R$, de genre $\geqq 2$, de $C_{0}-k$. Soit $V$ un voisinage de $\bar{R}$ dans $M-k$ tel que $C \cap V$ soit connexe et non-compact. Prenons enfin un tube $T=T(\bar{R})$ dans $V$ le long de $\bar{R}$ muni d'une rétraction holomorphe $\varphi: T \rightarrow \bar{R}$ (cf. le Lemme 1). Puisque $b T$ (cf. le Lemme 1) est compact et $b T \cap C=\varnothing$, il existe un rayon $\rho>0$ tel que $f\left(\Delta_{\rho}^{*}\right) \cap b T=\emptyset . \quad$ (Rappeler: $C=f(0)$ ). Soit $U$ l'intérieur de $T$, et considérons $G=f^{-1}(U)$ et l'application composée $g=\varphi \circ f_{\mid G}: G \rightarrow R$. On a alors $g(\zeta) \in \partial R$ pour tout $\zeta \in \partial G \cap \Delta_{\rho}^{*}$; par suite, $g(0 \mid \partial G-\{0\}) \subset \partial R$. D'antre part, de l'hypothèse $f(0)=C$, on a $g(0 \mid G)=\bar{R}$. Donc, d'après le théorème d'Ohtsuka que nous venons de citer, $R=g(0 \mid G)-g(0 \mid \partial G-\{0\})$ est de genre $\leqq 1$, ce qui est une contradiction. Donc, toute composante irréductible de $C$ est de genre $\leqq 1$.

C. Q.F.D.

(6) Nous avons modifié ici sa notation légèrement. 


\section{Chapitre II. —Cas où $f(0)$ Est une Courbe Analytique}

\section{Compacte dans une Surface Complexe}

4. Courbes de valeurs exceptionnelles et $l(\tilde{\boldsymbol{P}})$. Soit $S$ une surface complexe normale (c'est-à-dire, un espace analytique complexe normal de dimension complexe 2), et $f$ une application holomorphe de $\Delta^{*}: 0<|z|<1$ dans $S$. On supposera dans ce numéro et les deux suivants $\left(n^{\text {os }} 5,6\right)$ que

$$
C=f(0 ; S)
$$

est une courbe analytique compacte dans $S$.

Soit $k$ la réunion de l'ensemble $k_{0}$ des points singuliers de $S$ et de l'ensemble des points singuliers de $C$ dans $S-k_{0} ; k$ est alors discret. D'après $F$. Hirzebruch [6], en faisant la réduction de singularités de $S$ et de $C$, on peut trouver une surface complexe non-singulière $\tilde{S}$ et une application holomorphe propre $\mu$ de $\widetilde{S}$ sur $S$ telles que $\left.\mu\right|_{\tilde{S}-\mu^{-1}(k)}$ soit un isomorphisme analytique de $\widetilde{S}-\mu^{-1}(k)$ sur $S-k$ et que tout point singulier de $\mu^{-1}(C)$ soit un point double ordinaire. On voit aisément qu'il y a une application holomorphe $\tilde{f}$ de $\Delta^{*}$ dans $\tilde{S}$ telle que $f=\mu \circ \tilde{f}$. Puisque $\widetilde{C}=\tilde{f}(0 ; \tilde{S}) \subset \mu^{-1}(C), \tilde{C}$ est aussi, d'après la Proposition 3, une courbe analytique compacte, mais n'ayant, comme singularité, que des points doubles ordinaires.

On supposera donc dans ce qui suit que $S$ est non-singulière, et que les singularités de $C=f(0 ; S)$ sont des points doubles ordinaires (s'il y en a).

Soit $C_{0}$ une composante irréductible de $C$. On désignera par $\widetilde{C}_{0}$ son modèle non-singulière (ou la normalisée de $C_{0}$ ) et $\pi: \widetilde{C}_{0} \rightarrow C_{0}$ la projection. D'après l'hypothèse ci-dessus, $\pi^{-1}(P)$ a deux points pour les points singuliers $P$ de $C_{0}$; en dehors de ces points, $\pi$ est biunivoque. Considérons un point $\widetilde{P}$ de $\widetilde{C}_{0}$, et prenons un disque $\delta=\delta(\widetilde{P})$ de centre $\widetilde{P}$ sur $\widetilde{C}_{0}$ (c'est-à-dire, un ouvert $\delta$ défini par $|w|<r(<1)$ dans un voisinage de coordonnée locale $|w|<1$ de $\widetilde{C}_{0}$ en $\widetilde{P}, w(\widetilde{P})=0)$ de façon que $\pi(\bar{\delta}-\widetilde{P})$ ne contienne aucun point singulier de $C$, où $\delta=\delta \cup \partial \delta$. Posons

$$
C(\delta)=\text { la composante connexe de } C-\pi(\partial \delta) \text { contenant } \pi(\widetilde{P}) \text {. }
$$

Si $\pi(\widetilde{P})$ est un point régulier de $C$, on a $C(\delta)=\pi(\delta)$.

Définition 1. On dira que $C(\delta)$ est de type $(l, q)$, si les conditions suivantes sont remplies : 
1. $(l, q)$ est une paire d'entires positifs premiers entre eux tels que $0<q$ $<l$, ou bien $(l, q)=(1,0)$;

2. $\Theta=C(\delta)-\pi(\delta-\tilde{P})$ est une courbe compacte et exceptionnelle dans $S$, ou bien $\Theta=\pi(\widetilde{P})$ (dans ce cas-ci, on posera $(l, q)=(1,0)$ );

3. si l'on désigne par $\tau: S \rightarrow S_{*}=S / \Theta$ la modification analytique obtenue par la contraction de $\Theta$, le point $\tau(\Theta)$ a un voisinage $U_{*}$ analytiquement isomorphe à la normalisée $B(l, q)$ de l'hypersurface définie par l'équation: $t^{l}=$ $x^{l-q} y$ dans le tricylindre: $|x|<1,|y|<1,|t|<1$ et, par cette isomorphisme, $\tau(\pi(\delta))$ (resp. $\tau(\Theta))$ correspond à l'axe: $y=t=0$ (resp. à l'origine $0: x=y=t=0$ ).

$\left(\tau(\Theta)\right.$ est un point singulier normal de $S_{*}$, sauf le cas $l=1$ où $S_{*}$ est nonsingulière.)

Remarque 1. - Supposons qu'on ait $C(\delta)$ de type $(l, q)$ tel que $\Theta \neq \pi(\widetilde{P})$ et (faiblement) minimale au sens siuvant:

(M) Il n'y a aucune composante irréductible de $C(\delta)$ exceptionnelle de première espèce $^{(7)}$ et contenant deux au plus de points singuliers de $C(\delta)$.

On a alors, d'après H. Hopf [7], $l \neq 1$. De plus d'après F. Hirzebruch [6], toute composante irréductible $\Theta_{i}(i=1,2, \ldots, r)$ de $C(\delta)$ excepté $\pi(\delta)$ est compacte, non-singulière, de genre zéro, avec le nombre d'intersection $\left(\Theta_{i}, \Theta_{i}\right)$ $=-n_{i}$, où

$$
l / q=n_{1}-\frac{1}{n_{2}-{ }^{-\frac{1}{n_{r}}}},\left(n_{i} \geqq 2\right),
$$

et le graphe associé à $C(\delta)$ est de la forme suivante:

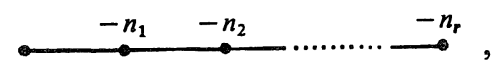

où les points - représentent de gauche à droite $\pi(\delta), \Theta_{1}, \Theta_{2}, \ldots, \Theta_{r}$ et les lignes - les points d'intersections de ces composantes.

Réciproquement, si $C(\delta)$ a cette forme-ci, $C(\delta)$ est de type $(l, q)$. En effet, si $r=1$, c'est le résultat de $H$. Grauert $\left([5], \S 4, \mathrm{n}^{\circ} 8\right)^{(8)}$; pour le cas où $r \geqq 2$, voir par exemple, E. Brieskorn [1].

(7) On dit qu'une courbe analytique irréductible $C$ est exceptionnelle de première espèce, si elle est compacte, non-singulière, de genre zéro et le nombre d'intersection $(C \cdot C)=-1$.

(8) On peut le montrer également, en appliquant le résultat de K. Kodaira et de D. C. Spencer [13] de façon analogue à K. Kodaira [11], II, Appendix. 
Remarque 2. - Soit $B(l, q)$ l'hypersurface normalisée considérée dans la condition 3 de la Définition 1 ci-dessus. Alors, pour toute application holomorphe $h$ d'un voisinage du disque fermé $\bar{\Delta}:|z| \leqq 1$ dans $B(l, q)$ vérifiant:

a) $h(\partial \Delta) \subset\{P \in B(l, q)|0<| x(P) \mid<1\}$;

b) $\frac{1}{2 \pi i} \int_{|z|=1} d \log x(h(z))=\lambda \neq 0$;

c) 1'origine $(0,0,0) \notin h(\Delta)$,

on a l'inégalité $l \leqq \lambda$. (En effet, la fonction $x=x(P)$ sur $B(l, q)$ prend les zéros d'ordre $l$ sur l'axe $[x=0] \cap B(l, q)$ moins l'origine; par suite, les zéros de la fonction $x(h(z))$ dans $\Delta$ sont tous d'ordre $\geqq l$.)

Définition 2. Si $E$ est une courbe analytique dans un ouvert $U$ de $S$ telle que le nombre des points de l'image réciproque $f^{-1}(E)$ dans $\Delta^{*}$ soit fini, $E$ sera appelée courbe analytique de valeurs exceptionnelles de $f$ (dans $U$ ). Soit $E(\delta)$ la réunion dans $S$ de toutes les courbes analytiques connexe de valeurs exceptionnelles $E$ de $f$ contenant au moins un point de $\pi(\delta)$ telles que $E \cap \pi(\partial \delta)=\varnothing$. On définit:

$$
l(\delta)=\left\{\begin{array}{lll}
1, & \text { si } \quad E(\delta) \subset C(\delta) & \text { et } \quad E(\delta) \neq C(\delta) \\
l, & \text { si } E(\delta)=C(\delta) & \text { et de type }(l, q) \\
\infty, & \text { autrement }
\end{array}\right.
$$

et $l(\widetilde{P})=\inf _{\tilde{P} \in \delta} l(\delta)$.

Sous ces notations, nous allons montrer le

Théorème 4. $\sum_{\widetilde{P} \in \tilde{C}_{0}}\left(1-\frac{1}{l(\widetilde{P})}\right) \leqq 2-2 g$, ò̀ g est le genre de $\widetilde{C}_{0}$.

On déduit aussitôt de ce théorème les propositions suivantes: $1^{\circ}$ toute composante irréductible de $C$ est de genre $\leqq 1$ (qu'on le sait déjà par le Théorème 3$) ; 2^{\circ}$ si $\widetilde{C}_{0}$ est elliptique $(g=1)$, on a $l(\widetilde{P}) \equiv 1$ sur $\widetilde{C}_{0} ; 3^{\circ}$ si $\widetilde{C}_{0}$ est rationnelle $(g=0)$, on a $l(\widetilde{P})=1$ excepté quatre points $\widetilde{P}_{j}$ au plus de $\widetilde{C}_{0}$ et on a $\sum_{1}^{4}\left(1-\frac{1}{l\left(\widetilde{P}_{j}\right)}\right) \leqq 2$.

La démonstration du Théorème 4 sera achevée à la fin du $n^{\circ} 6$.

5. Ensemble limite d'une suite d'images analytiques du disque fermé $\bar{\Delta}:|\boldsymbol{z}| \leqq \mathbb{1}$. Nous allons commencer par préparer quelques lemmes:

Lemme 2. Soit $\Theta$ une courbe irréductible, rationnelle ${ }^{(9)}$ et non-singulière

(9) Une courbe analytique compacte de genre 0 sera appelée courbe rationnelle. 
dans une surface analytique complexe non-singulière $S$ avec le nombre d'inter section $(\Theta \cdot \Theta) \geqq 0$, et $\sigma_{1}, \sigma_{2}, \ldots, \sigma_{n}, \ldots$, une suite de courbes analytiques noncompactes dans un voisinage $U$ de $\Theta$, ayant au moins un point d'accumulation sur $\Theta$. Il existe alors un entier $n>0$ tel que $\sigma_{n} \cap \Theta \neq \varnothing$.

En effet, si $(\Theta \cdot \Theta)=0, \Theta$ étant non-singulière et rationnelle, on peut trouver, d'après K. Kodaira-D. C. Spencer [13] (cf. K. Kodaira [11], II, Appendix), une fonction holomorphe $h$ dans un voisinage $V$ de $\Theta$ qui s'annule seulement sur $\Theta$. Prenons un nombre réel $\varepsilon>0$ tel que l'on ait $V_{\varepsilon}=\{P \in V \mid$ $|h(P)|<\varepsilon\} \Subset U \cap V$. D'après l'hypothèse, il existe un entier $N>0$ tel que $\sigma_{N}^{\prime}=\sigma_{N} \cap V_{\varepsilon} \neq \emptyset$. Or, si $\sigma_{N} \cap \Theta=\emptyset, 1 / h$ est holomorphe sur $\sigma_{N}^{\prime}$ et $|1 / h| \equiv 1 / \varepsilon$ sur le bord $\partial \sigma_{N}^{\prime}$; on a donc, $|1 / h| \leqq 1 / \varepsilon$ sur $\sigma_{N}^{\prime}$, ce qui est impossible, puisque $\sigma_{N}^{\prime} \subset V_{\varepsilon}$. Donc, $\sigma_{N} \cap \Theta \neq \emptyset$.

Puis, si $(\Theta \cdot \Theta)=m>0$, on prend un point d'accumulation $P$ de la suite $\left\{\sigma_{n}\right\}$ et $m$ points distincts $Q_{1}, Q_{2}, \ldots, Q_{m}$ de $\Theta-P$. Soit $\widetilde{S}$ la surface obtenue par $m$ transformations quadratiques ( $\sigma$-processus) en ces points $Q_{j}$, et $\widetilde{\Theta}$ (resp. $\tilde{\sigma}_{n}$ ) 1'image de $\Theta$ (resp. de $\sigma_{n}$ ) dans $\tilde{S}$. On a alors $(\widetilde{\Theta} \cdot \widetilde{\Theta})=0$ et la suite $\left\{\tilde{\sigma}_{n}\right\}$ s'accumule au point $P \in \widetilde{\Theta}$. On a donc, d'après ce qui précéde, un entier $N>0$ tel que $\tilde{\sigma}_{N} \cap \widetilde{\Theta} \neq 0$, ce qui entraine $\sigma_{N} \cap \Theta \neq \varnothing$ et achève la démonstration.

Surfaces de Riemann et revêtements ramifiés. - Une variété analytique complexe de dimension complexe pure 1 sera appelée surface de Riemann. Soient $\sigma, R$ deux surfaces de Riemann, où $R$ est connexe; $\phi: \sigma \rightarrow R$ une application holomorphe non-dégénérée et propre ${ }^{(10)}$. Alors, $\phi$ est surjective. (En effet, $\phi$ étant non-dégénérée, $\phi(\sigma)$ est ouvert; d'autre part, puisque $\phi$ est propre, $\phi(\sigma)$ est fermé; $R$ étant connexe, il s'en suit que $\phi(\sigma)=R$.) Nous disons que $\sigma$ est un revêtement (analytiquement) ramifié de $R$ à un nombre fini de feuillets par rapport à la projection $\phi$, ou simplement un revêtement ramifié fini de $R$.

On dira qu'une surface de Riemann $R$ est finie, si elle est analytiquement isomorphe à une surface obtenue d'une surface de Riemann compacte $\hat{R}$ par l'exception d'un nombre fini de disques fermés disjoints les uns les autres; on désignera par $n(R)$ le nombre de ces disques exclus de $\hat{R}$. Alors, le genre $g(R)$ de $R$ est égal à celui de $\hat{R}$, et la caractéristique (au sens d'Ahlfors) de $R$ est donnée par:

$$
e(R)=e(\widehat{R})+n(R)=2 g(R)-2+n(R) .
$$

(10) Une application $\phi: \sigma \rightarrow R$ sera dite non-dégénérée, si $\phi^{-1}(y)$ est discret pour tout $y \in R$; propre, si $\phi^{-1}(k)$ est compacte pour tout compact $k$ de $R$. 
Lemme 3. Si $\sigma$ est un revêtement ramifié fini et de genre zéro d'une surface de Riemann finie $R$, alors $R$ est aussi de genre zéro.

En effet, soient $m$ le nombre de feuillets de $\sigma$ et $v$ l'ordre de ramification total de $\sigma$; on a, d'après la formule d'Hurwitz, $e(\sigma)=m \cdot e(R)+v \geqq m \cdot e(R)$. Or, $n(\sigma) \leqq m \cdot n(R)$. On a donc, $m \cdot e(\hat{R}) \leqq e(\hat{\sigma})<0$; d'où $e(\hat{R})<0 . \quad R$ est donc de genre zéro.

Soit maintenant $R=\Delta_{w}-\bigcup_{i=1}^{\alpha} \delta_{i}$, où $\Delta_{w}$ est le disque unité $|w|<1$ dans le plan $w$, et $\left\{\delta_{i}\right\}$ sont $\alpha \geqq 1$ disques analytiques fermés ${ }^{(11)}$ disjoints dans $\Delta_{w}$. Soit $\gamma$ un compact dans le disque unité $\Delta_{z}:|z|<1$ dont le bord $\partial \gamma$ se décompose en un nombre fini de courbes analytiques réelles (fermée), non-singulières et disjointes. Posons $\sigma=\Delta_{z}-\gamma$. Supposons enfin qu'il y ait une application holomorphe $\phi$ d'un voisinage de $\bar{\sigma}$ sur un voisinage de $\bar{R}$ vérifiant les conditions suivantes:

1. $\phi_{\mid \sigma}: \sigma \rightarrow R$ est propre;

2. $\phi^{-1}\left(\partial \Delta_{w}\right)=\partial \Delta_{z}$;

3. Pour toute composante simplement connexe $\gamma_{v}$ de $\gamma$, la multiplicité de la courbe fermé $\partial \gamma_{v}$ (par rapport à $\phi$ ) sur $\phi\left(\partial \gamma_{v}\right)=\partial \delta_{i(v)}$ est $\geqq 2$ (c'est-à-dire, $\phi_{\mid \partial \gamma_{\nu}}$ n'est pas injective).

Lemme 4. Dans ces hypothèses, on a: $\alpha\left(=\right.$ le nombre des disques $\left.\delta_{i}\right)=1$.

En effet, soit $m(\geqq 2)$ le nombre de feuillets de $\sigma$ sur $R$. Comme on l'a déjà vu dans la preuve du Lemme 3 , on a $m \cdot e(R) \leqq e(\sigma)$. D'autre part, $e(R)$ $=\alpha-1$ et $e(\sigma)=e\left(\Delta_{z}\right)-e(\gamma) \leqq-1+p$, où $p$ est le nombre des composantes simplement connexes de $\gamma$. Or, d'après les hypothèses 2 et 3 , on a $p \leqq \alpha \cdot \frac{m}{2}$. Par conséquent, $m(\alpha-1) \leqq-1+\alpha \cdot \frac{m}{2}$, d'où $\alpha / 2 \leqq 1-\frac{1}{m}<1 ; \alpha$ étant un entier $\geqq 1$, on a $\alpha=1$.

C. Q.F.D.

Nous allons maintenant prouver la

Proposition 4. Soient $S$ une surface complexe non-singulière, $C$ une courbe analytique compacte sur $S$ dont les points singuliers sont des points doubles ordinaires, et $P_{0}$ un point double de $C$. Soit $\Gamma:|x| \leqq 1,|y| \leqq 1^{(12)}$ un dicylindre fermé de coordonnées locales $x, y$ de $S$ en $P_{0}$ tel que $\Gamma \cap C=\{P \in \Gamma \mid x(P) \cdot y(P)$

(11) Nous appellerons un ouvert (resp. fermé) contractile limité par une seule courbe analytique réelle fermée et non-singulière (sur une surface de Riemann) disque analytique (resp. disque analytique fermé).

(12) On suppose que $x$ et $y$ sont holomorphes au voisinage de $\Gamma$. 
$=0\}$; posons

$$
\begin{aligned}
& K=\left\{P \in \Gamma|| x(P) \mid<s_{0}, y(P)=0\right\} \quad\left(0<s_{0}<1\right), \\
& C(K)=\text { la composante connexe de } C-\partial K \text { contenant } P_{0} .
\end{aligned}
$$

Et supposons qu'on ait une suite d'applications holomorphes $f_{1}, f_{2}, \ldots, f_{n}, \ldots, d u$ voisinage de $\bar{\Delta}:|z| \leqq 1$ dans $S$ vérifiant les conditions suivantes $\left(0<s_{0}<s<1\right)$ :

(1) $f_{n}(\partial \Delta) \subset\left\{P \in \Gamma\left|s_{0} \leqq\right| x(P) \mid \leqq s\right\} \quad$ et $\frac{1}{2 \pi i} \int_{|z|=1} d \log x\left(f_{n}(z)\right)=\lambda$ (indépendant de $\left.n\right)>0$;

(2) $f_{n}(\bar{\Delta}) \cap C(K)=\emptyset$;

(3) $f_{n}(\bar{\Delta}) \cap\{P \in \Gamma|s<| x(P) \mid \leqq 1\}=\varnothing$;

(4) $\lim _{n \rightarrow \infty} f_{n}(\bar{\Delta}) \subset C_{\text {. }}^{(13)}$

Alors, $C(K)$ est de type $(l, q)$ avec $l \leqq \lambda$ et $C(K) \subset \lim _{n \rightarrow \infty} f_{n}(\bar{\Delta})$.

Démonstration. $C(K)$ est une courbe analytique dans $S-\partial K$. Soient $K$, $\Theta_{1}, \Theta_{2}, \ldots, \Theta_{r}$ les composantes irréductibles de $C(K)$ numérotées de telle manière que $\Theta_{1} \cap K \neq \varnothing, \Theta_{k} \cap \bigcup_{i=1}^{k-1} \Theta_{i} \neq \varnothing(k=2, \ldots, r)$. Posons $\Theta=\bigcup_{i=1}^{r} \Theta_{i}$. D'après les Remarques 1 et 2 à la Définition $1\left(n^{\circ} 4\right)$, il suffira de prouver trois choses suivantes: (a) Toute composante $\Theta_{i}(i=1,2, \ldots, r)$ est compacte, nonsingulière, rationnelle et de nombre d'intersection $\left(\Theta_{i}, \Theta_{i}\right)<0$; (b) $C(K)$ supposé minimal au sens $(\mathrm{M})$ de la Remarque $1\left(n^{\circ} 4\right)$, son graphe est linéaire:

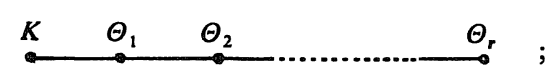

et (c) $\quad C(K) \subset \lim _{n \rightarrow \infty} f_{n}(\bar{\Delta})$.

$1^{\circ} \quad$ A cet effet, prenons un dicylindre fermé $\Gamma_{\mu}:\left|x_{\mu}\right| \leqq 1,\left|y_{\mu}\right| \leqq 1^{(12)}$ de coordonnées locales $x_{\mu}, \mathrm{y}_{\mu}$ de $S$ à chaque point singulier $P_{\mu}(\mu=0,1, \ldots, m)$ de $C(K)$, disjoint l'un de l'autre et tel que l'on ait $\Gamma_{\mu} \cap C=\left\{P \in \Gamma_{\mu} \mid x_{\mu}(P) \cdot y_{\mu}(P)=0\right\}$, où $\left(\Gamma_{0}, x_{0}, y_{0}\right)=(\Gamma, x, y)$. Soit $s<s_{1}<1$, et $R=\Theta-\bigcup_{\mu=0}^{m} \Gamma_{\mu}\left(s_{1}\right)$, où $\Gamma_{\mu}(\rho)=\{P \in$ $\left.\Gamma_{\mu}|| x_{\mu}(P)|\leqq \rho,| y_{\mu}(P) \mid \leqq \rho\right\}$ pour $0<\rho<1$. Prenons un tube $T$ le long de $\bar{R}$ (cf. Lemme $1, \mathrm{n}^{\circ} 3$ ) dans $S-\left\{P_{\mu}\right\}$, muni d'une rétraction holomorphe $\varphi: T \rightarrow \bar{R}$ et tel que:

(5) $\Gamma_{\mu} \cap T \subset\left\{P \in \Gamma_{\mu}|s<| x_{\mu}(P)|\leqq 1,| y_{\mu}(P) \mid<s\right\}$

$$
\cup\left\{P \in \Gamma_{\mu}|| x_{\mu}(P)|<s, s<| y_{\mu}(P) \mid \leqq 1\right\} ;
$$

(13) $\lim _{n \rightarrow \infty} f_{n}(\bar{\triangle}):=\bigcap_{n=1}^{\infty} \overline{\bigcup_{k=n}^{\infty} f_{k}(\bar{J})}$ (définition). 
(6) $\varphi^{-1}(\partial R) \subset \bigcup_{\mu=0}^{m}\left\{\Gamma_{\mu}-\Gamma_{\mu}(s)\right\}$,

et désignons par $R_{i}$ (resp. $T_{i}$ ) la composante connexe de $R$ sur $\Theta_{i}$ (resp. de $T$ contenant $R_{i}$ ). De l'hypothèse (4), il existe un entier $N \geqq 1$ tel que pour tout $n \geqq N$ l'on ait:

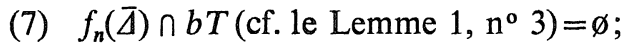

(8) $f_{n}(\bar{\Delta}) \cap H_{\mu}(\varepsilon)=\varnothing$ pour $\mu=0,1, \ldots, m$;

où $H_{\mu}(\varepsilon)=\left\{P \in \Gamma_{\mu}|\varepsilon \leqq| x_{\mu}(P)|\leqq 1, \varepsilon \leqq| y_{\mu}(P) \mid \leqq 1\right\}$ et $\varepsilon$ est un nombre réel compris dans $0<\varepsilon<s_{0}$ tel que l'on ait

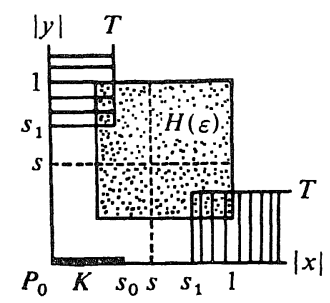

(9) $\Gamma_{\mu} \cap b T \subset H_{\mu}(\varepsilon)$.

Fixons un $n \geqq N$ pour le moment. Alors, $f_{n}(\bar{\Delta})$ est inclu dans $W=\bigcup_{\mu=0}^{m}\left[\Gamma_{\mu}\right.$ $\left.-H_{\mu}(\varepsilon)\right] \cup V$, où $V$ est l'intérieur de $T$. Considérons l'image réciproque $D$ $=f_{n}^{-1}(V)$ dans $\Delta:|z|<1$. D'après l'hypothèse (1), on a $D \Subset \Delta$. En variant $s_{1}$ légèrement (s'il est nécessaire), on peut supposer que le bord $\partial D$ de $D$ se décompose en un nombre fini de courbes analytiques réelles fermées, disjointes et nonsingulières (pour tout $n \geqq N$ ). Soient $\left\{D_{k}\right\}$ les composantes connexes de $D$, et désignons par $R_{i(k)}$ la composante connexe de $R$ contenant $\varphi\left(f_{n}\left(D_{k}\right)\right)$. Alors, d'après la condition (7), $D_{k} \stackrel{\varphi \circ f_{n}}{\longrightarrow} R_{i(k)}$ est propre.

(10) $D_{k}$ est un revêtement ramifié fini de $R_{i(k)}$ par rapport à la projection $\varphi_{k}=\varphi \circ f_{n \mid D_{k}}$.

D'autre part, si $G_{0}=f_{n}^{-1}(\Gamma-V)$ était simplement connexe (c'est-à-dire $\left.G_{0}=\bar{\Delta}\right)$, la fonction $x(f(z))$ devrait avoir des zéros dans $\Delta$, d'après l'hypothèse (1), et on aurait $f_{n}(\Delta) \cap \Theta_{1} \neq \varnothing$ contrairement à (2); si en suite $\bar{\Delta}-D$ possédait une composante simplement connexe $G_{j}\left(\neq G_{0}\right)$, l'image $\sigma=f_{n}\left(G_{j}\right)$ se trouverait dans un $\Gamma_{\mu}-V$ et son bord unique $\partial \sigma\left(=f_{n}\left(\partial G_{j}\right)\right)$ dans $\varphi^{-1}(c), c$ étant le cercle $\left|x_{\mu}\right|=s_{1}$ sur l'axe $x_{\mu}$ ou bien $\left|y_{\mu}\right|=s_{1}$ sur l'axe $y_{\mu}$; soit $c$ sur l'axe $x_{\mu}$ pour fixer l'idée; on vérifierait aisément $(1 / 2 \pi i) \int_{\partial \sigma} d \log x_{\mu} \neq 0$; d'où $\sigma \cap\left(1\right.$ 'axe $\left.y_{\mu}\right) \neq \varnothing$ contrairement à (2). Donc,

(11) $\Delta-D$ ne possède aucune composante simplement connexe.

De la même manière, puisque $f_{n}(\Delta) \cap K=\emptyset$, tenant compte de (3) et de (8), on obtient la proposition:

(12) $G_{0}=f_{n}^{-1}(\Gamma-V)$ (dont le bord contient $\left.\partial \Delta\right)$ est connexe.

Maintenant, considérons un couple $\left(\gamma_{1}, A\right)$ d'une composante connexe $\gamma_{1}$ 
(courbe fermée) de $\partial D$ telle que le côté intérieur de $\gamma_{1}$ appartienne à $D$ (par exemple, une composante de $\partial G_{0}$ dans $\Delta$ ) et d'une suite

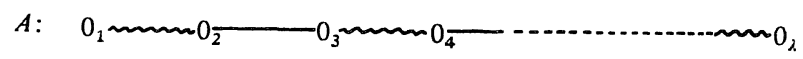

de composantes connexes $0_{\lambda}$ de $\partial R$, où $0_{1}=\varphi \circ f_{n}\left(\gamma_{1}\right)$, où mm et - apparaissent alternativement et $0_{\lambda} \sim m 0_{\lambda+1}$ (resp. $0_{\lambda}-0_{\lambda+1}$ ) signifie que $0_{\lambda}$ et $0_{\lambda+1}$ sont sur la même $\bar{R}_{i}$ (resp. $\Gamma_{\mu}$ ). Alors, d'après (10) et (11), nous obtenons la proposition suivante:

(13) Pour tout couple $\left(\gamma_{1}, A\right)$, il existe une suite

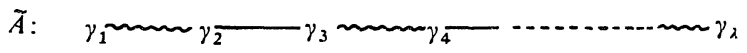

de composantes connexes $\gamma_{\lambda}$ de $\partial D$ telle que l'on ait

$$
\varphi \circ f_{n}\left(\gamma_{\lambda}\right)=0_{\lambda} \text { pour tout } \lambda \text {, }
$$

ò̀ $\gamma_{\lambda} \sim \cdots \gamma_{\lambda+1}$ (resp. $\gamma_{\lambda}-\gamma_{\lambda+1}$ ) signifie que $\gamma_{\lambda}$ et $\gamma_{\lambda+1}$ sont sur la même $D_{k}$ (resp. la même composante connexe $G_{j}$ de $\Delta-D$ ).

Puisque les $m$ et - apparaissent alternativement dans la suite $\tilde{A}, \gamma_{\lambda+1}$ se trouve dans l'intérieur de la courbe $\gamma_{\lambda}$; en particulier, $\gamma_{1}, \gamma_{2}, \ldots, \gamma_{\lambda}, \ldots$ sont différentes les unes des autres. Par conséquent, la longueur de la suite $(A)$ est nécessairement finie, ce qui entraîne que tout composante $\Theta_{i}(i=1,2, \ldots, r) d e$ $\Theta$ est non-singulière et le graphe associé à $C(K)$ est contractile (un arbre).

De plus, si l'on prend une composante de $\partial G_{0}$ dans $\Delta$ comme $\gamma_{1}$, il résulte de (13) que l'application $D \stackrel{\varphi \circ f_{n}}{\longrightarrow} R$ est surjective $(n \geqq N)$; on en déduit deux choses: d'une part, d'après le Lemme 3 , toute $\Theta_{i}(i=1,2, \ldots, r)$ est de genre zéro; d'autre part, en faisant $n \rightarrow \infty$, on a

(14) $R \subset \lim _{n \rightarrow \infty} f_{n}(\Delta)$;

par suite, d'après l'hypothèse (3), on a $\bar{\Theta} \cap \partial K=\emptyset$, et $\Theta$ est donc compacte.

Nous avons ainsi vu que chaque $\Theta_{i}$ est une courbe compacte, rationnelle et non-singulière dans $S$. Dans $U=S-\left\{P \in \Gamma\left|s_{0} \leqq\right| x(P) \mid \leqq s\right\}$, on a une suite de courbes analytiques $\left\{\sigma_{n}=f_{n}(\Delta) \cap U, n=1,2, \ldots\right\}$ telle que l'on ait $\sigma_{n} \cap \Theta_{i}=\varnothing$ pour tout $n$ et $\Theta_{i} \cap \lim _{n \rightarrow \infty} \sigma_{n} \neq \varnothing$. On a donc, d'après le Lemme $2,\left(\Theta_{i} \cdot \Theta_{i}\right)<0$ pour tout $i$.

$2^{\circ}$ Après avoir prouvé (a), il nous reste à prouver (b), c'est-à-dire que, $C(K)$ supposé minimal, le graphe $\mathfrak{g}$ associé à $C(K)$ est linéaire. Nous avons déjà vu dans $1^{\circ}$ que $\mathfrak{g}$ est un arbre. Supposons que $\mathfrak{g}$ ne soit pas linéaire: $\mathfrak{g}$ posséderait alors un point à $\alpha+1$ branches $(\alpha \geqq 2)$ tel que, exceptée la branche 
$\mathrm{g}_{0}$ qui contienne le point correspondant à la composante $K$ de $C(K)$, toute autre branch $\mathfrak{g}_{v}(v=1,2, \ldots, \alpha)$ soit linéaire:

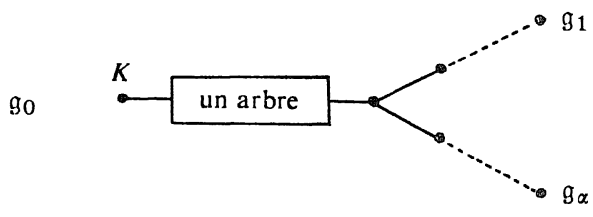

Soit $\Theta_{i_{0}}$ la composante de $\Theta$ correspondant à ce point, et $W_{0}$ la composante connexe de $W-T_{i_{0}}$ contenant $K$, où $W=\cup_{\mu=0}^{m}\left[\Gamma_{\mu}-H_{\mu}(\varepsilon)\right] \cup V . \quad R_{i_{0}}$ s'obtiendrait de $\Delta_{w}=\Theta_{i_{0}}-\bar{W}_{0}$ analytiquement isomorphe à un disque $|w|<1$ dans le plan $w$ par l'exclusion de $\alpha(\geqq 2)$ disques analytiques fermés disjoints. Or, on déduit aussitôt des propositions (13) et (12) de $1^{\circ}$ ci-dessus, que $f_{n}^{-1}\left(W_{0}\right)(n \geqq N)$ ne possède aucune composante simplement connexe, de sorte que le complément $\Delta-\overline{f_{n}^{-1}\left(w_{0}\right)}$ possède au moins une composante simplement connexe $F$. D’après (10), $\sigma=F \cap f_{n}^{-1}\left(V_{i_{0}}\right)$ est un revêtement ramifié fini de $R_{i_{0}}$ par rapport à la projection $\phi=\varphi \circ f_{n \mid \bar{\sigma}}$, et on a $\phi^{-1}\left(\partial \Delta_{w}\right)=\partial F$. D'autre part, compte tenue de la proposition (a) déjà prouvée et de la Remarque 1 ( $\left.\mathrm{n}^{\circ} 4\right)$, la partie de $\Theta$ correspondant à chaque sous-graphe linéaire $g_{v}(v=1,2, \ldots, \alpha)$ serait de type $\left(l_{v}, q_{v}\right)$ avec $l_{\nu} \geqq 2$; de la sorte, d'après la Remarque 2 du $\mathrm{n}^{\circ} 4$, le bord $\partial \gamma$ de chaque composante simplement connexe $\gamma$ de $F-\sigma$ serait multivalent sur $\phi(\partial \gamma)$, ce qui contredit le Lemme 4, car on a supposé $\alpha \geqq 2$. Donc, $\mathfrak{g}$ est linéaire (la proposition (b)). Quant'à $C(K) \subset \lim _{n \rightarrow \infty} f_{n}(\Delta)$, il suffit de faire $\varepsilon \rightarrow 0$ dans la condition (8), tenant compte de (14). $\quad$ La Proposition 4 est démontrée.

Lemme 5. Soit $E$ une courbe analytique dans une surface complexe non-singulière $U$, et considérons une suite de courbes analytiques $\sigma_{n}$ dans $U$ telles que $\sigma_{n} \cap E=\varnothing$. Alors, l'ensemble

$$
\left(\lim _{n \rightarrow \infty} \sigma_{n}\right) \cap E
$$

n'a pas de point isolé. $\quad\left(\right.$ Où $\lim _{n \rightarrow \infty} \sigma_{n}=\bigcap_{n=1}^{\infty} \overline{\bigcup_{v=n}^{\infty} \sigma_{v}}$.)

En effet, supposons que $P_{0}$ soit un point isolé de $\Sigma=\left(\lim \sigma_{n}\right) \cap E$. Prenons un voisinage $V \Subset U$ de $P_{0}$ tel que l'on ait $\bar{V} \cap \Sigma=\left\{P_{0}\right\}$ et que $E$ s'écrite $f=0$ dans un voisinage $V^{\prime}$ de $\bar{V}$ à l'aide d'une fonction holomorphe $f$. $\lim \sigma_{n}$ étant fermé et $E \cap \partial V$ compact, il existe un voisinage $W\left(\Subset V^{\prime}\right)$ de $E \cap \partial V$ tel que 
$\left(\lim \sigma_{n}\right) \cap W=\varnothing . \quad$ Soit $\lambda$ le minimum des modules $|f(P)|$ pour $P \in(\partial V)-W(>0)$, et posons $D=\{P \in V|| f(P) \mid<\lambda\}$. On a $\partial D \cap\left\{P \in V^{\prime}|| f(P) \mid<\lambda\right\} \subset W$. Or, si l'on prend un entier $N$ suffisamment grand, on a $\sigma_{N} \cap \bar{W}=\emptyset$ et $\sigma_{N} \cap D \neq \emptyset$. Par conséquent, l'application $z=f_{\mid \sigma_{N} \cap D}: \sigma_{N} \cap D \rightarrow|z|<\lambda$ est propre, non-dégénérée, de sorte que surjective. Donc, f prend 0 à un point de $\sigma_{N}$, c'est-à-dire $\sigma_{N} \cap E$ $\neq \varnothing$, ce qui est contraire à l'hypothèse. Donc, $\Sigma$ n'a pas de point isolé.

C. Q.F.D.

Revenons maintenant à la situation du $\mathrm{n}^{\circ} 4$, et rappelons les $S, f: \Delta^{*} \rightarrow S$, $C=f(0 ; S), C_{0}, \pi: \widetilde{C}_{0} \rightarrow C_{0}, l(\delta), l(\widetilde{P})$, etc. . Soient $\widetilde{P}$ un point de $\widetilde{C}_{0}, \delta$ un disque analytique sur $\widetilde{C}_{0}$ de centre $\widetilde{P}$ et $\Gamma:|x|<1,|y|<1$ un voisinage de coordonnées locales $x, y$ de $S$ en $\pi(\widetilde{P})$ tel que:

1. $\pi(\bar{\delta}) \subset\left\{P \in \Gamma|| x(P) \mid<s_{0}, y(P)=0\right\} \quad\left(0<s_{0}<1\right)$;

2. $C \cap \Gamma \subset\{P \in \Gamma \mid x(P) \cdot y(P)=0\}$.

Cela posé, on a la

Proposition 5. Supposons qu'il existe un entier $m>0$ et une suite infinie de disques analytiques ${ }^{(11)}$ disjoints $\Delta_{1}, \Delta_{2}, \ldots, \Delta_{n}, \ldots$, dans $\Delta^{*}: 0<|z|<1$ tendant vers l'origine $z=0$ et vérifiant les conditions suivantes (pour un nombre réel $s$ compris dans $\left.s_{0}<s<1\right)$ :

a) $f\left(\partial \Delta_{n}\right) \subset\left\{P \in \Gamma\left|s_{0}<\right| x(P) \mid<s\right\}$;

b) $\frac{1}{2 \pi i} \int_{\partial \Delta_{n}} d \log x(f(z))=m$ (indépendant de $\left.n\right)>0$;

c) $f\left(\Delta_{n}\right) \cap\{P \in \Gamma|s<| x(P) \mid<1\}=\varnothing$.

Alors, on a $l(\widetilde{P}) \leqq m(<\infty)$.

Démonstration. - $1^{\circ}$ Montrons d'abord $E(\delta) \subset C(\delta)$. Pour cela, étant donnée une courbe analytique connexe $E$ de valeurs exceptionnelles de $f$ telle que $E \cap \pi(\delta) \neq \varnothing$ et $E \cap \pi(\partial \delta)=\varnothing$ dans un sous-domaine $U$ de $S$, nous avons à prouver $E \subset C(\delta)$. Soit $H_{\varepsilon}=\left\{P \in \Gamma\left|s_{0}-\varepsilon \leqq\right| x(P)|\leqq s+\varepsilon, \quad| y(P) \mid \leqq \varepsilon\right\}$, où $\varepsilon>0$ est assez petit pour que l'on ait $\pi(\bar{\delta}) \cap H_{\varepsilon}=\varnothing$. Il suffira de prouver $E-H_{\varepsilon} \subset$ $C(\delta)$, car cela entraîne, faisant $\varepsilon \rightarrow 0, E \subset C(\delta)$. On peut donc supposer à nouveau $U \cap H_{\varepsilon}=\varnothing$ et $E \cap H_{\varepsilon}=\varnothing$. Maintenant, puisque $\lim _{n \rightarrow \infty} f\left(\partial \Delta_{n}\right) \subset H_{\varepsilon / 2}$, il existe un entier $N>0$ tel que, pour tout $n \geqq N, \sigma_{n}=f\left(\Delta_{n}\right) \cap \stackrel{n \rightarrow \infty}{U}$ soit une courbe analytique de $U$.

D'après le théorème de $\mathrm{P}$. Thullen cité au $\mathrm{n}^{\circ} 3, \underset{n \geqq N}{\cup} \sigma_{n}$ étant une courbe analytique de $U-C, \Sigma=\lim _{n \rightarrow \infty} \sigma_{n}$ se compose de composantes irréductibles de 
$C \cap U$. Remarquons que $\Sigma$ contient $\pi(\delta) \cap U(\neq \varnothing)$. Or, d'après le Lemme 5 , $E_{i} \cap \Sigma$ n'est discret pour aucune composante irréductible $E_{i}$ de $E$. Comme $E \cap \Sigma \neq \varnothing$ et $E$ connexe, il s'en suit que $E \subset \Sigma$.

$2^{\circ}$ Après avoir prouvé $E(\delta) \subset C(\delta)$, il nous reste à prouver, pour le cas $E(\delta)=C(\delta)$, que $C(\delta)$ est de type $(l, q)$ avec $l \leqq m$. Or, cela résulte de la Proposition 4 , car les $\bar{\Delta}_{n}(n=1,2, \ldots)$ sont analytiquement équivalentes au disque unité fermé $\bar{\Delta}:|z| \leqq 1$ du plan d'une variable complexe $z$. La Proposition 5 se trouve démontrée.

6. Surfaces de recouvrements d'Ahlfors. Dans ce numéro, une surface de Riemann finie signifiera une surface de Riemann compacte, à bords réguliers ou sans bord, triangularisée. Soit $R$ une surface de Riemann finie, munie d'une métrique conforme $d s=\mu(z)|d z|$ à coefficient $\mu(z)$ strictement positif en tout point de $R$. Soit $F$ une surface de recouvrement finie(14) de $R$. On munit $F$ de la métrique $d \tilde{s}=\tilde{\mu}(z)|d z|$ induite de $d s$ par la projection. (Le coefficient de $d \tilde{s}$ s'annule aux points de ramifications de $F$ ). On posera, pour chaque arc régulier $\gamma$ sur $R$ (resp. $F$ ), $|\gamma|=\int_{\gamma} d s\left(\right.$ resp. $\left.\int_{\gamma} d \tilde{s}\right)$ et, pour chaque région $\Delta$ de $R$ (resp. de $F$ ), $|\Delta|=\iint_{\Delta} \mu(z)^{2} d x d y$ (resp. $\left.\iint_{\Delta}^{\gamma} \tilde{\mu}(z)^{2} d x d y\right)$, où $z=$ $x+\sqrt{-1} y$. Le théorème principal de $\mathrm{L}$. Ahlfors ${ }^{(15)}$ dit qu'il existe une constante $k>0$ dépendant seulement de $(R, d s)$ telle que

$$
e^{+}(F) \geqq M(F) \cdot e(R)-k \cdot L(F),
$$

où $e^{+}(F)=\max \{e(F), 0\}, e(F)$ et $e(R)$ étant les caractéristiques de $F$ et de $R$ respectivement ${ }^{(16)}, M(F)=\frac{|F|}{|R|}$ est la moyenne de nombre de feuillets de $F$ et $L(F)=|\partial F|$ la longueur de la frontière $\partial F$ de $F$ relative à $R$. Le second théorème de recouvrement de L. Ahlfors dit que, pour chaque arc régulier $\gamma$ sur $R$, il existe une constante $k^{\prime}>0$ dépendant seulement de $\gamma$ et de $(R, d s)$ telle que

$$
|M(F)-M(\gamma)|<k^{\prime} \cdot L(F),
$$

où $M(\gamma)=\mid$ la partie de $F$ au-dessus de $\gamma|/| \gamma \mid$ est la moyenne de nombre de

(14) 《inite covering surface》 dans [25], qui peut avoir des points de ramification et aussi des points frontières relatifs à $R$. Nous adoptons ici la terminologie 《surface de recouvrement $\rangle$ avec J. Dufrenoy (Ann. scient. Ec. Norm. Sup. (3) 58 (1941), 179-259).

(15) Pour la théorie d'Ahlfors de 《covering surfaces》 appliquée ci-dessous, voir par exemple [25], Chapter VI.

(16) La caractéristique $e(R)$ d'une surface de Riemann finie (triangularisée) $R$ ayant $f$ faces, a arêtes intérieurs et $s$ somments intérieurs est, par définition, $e(R)=-s+a-f$ (cf. $\left.\mathrm{n}^{\circ} 5\right)$. 
feuillets de $F$ au-dessus de $\gamma$. On remarquera que $\gamma$ peut se trouver sur le bord $\partial R$ de $R$.

Soit $F^{*}$ une surface de recouvrement infinie et à bords ${ }^{(17)}$ d'une surface de Riemann finie $R$. On dira qu'une suite croissante de sous-ensembles compacts $F_{1} \subset F_{2} \subset \cdots \subset F_{n} \subset \cdots$ de $F^{*}$ telle que $F^{*}=\bigcup_{n=1}^{\infty} F_{n}$ est une exhaustion régulière de $F^{*}$, si les deux conditions suivantes sont remplies:

$1^{\circ}$ chaque $F_{n}$ est une surface de Riemann finie;

$2^{\circ}$ on a $\lim _{n \rightarrow \infty} \frac{L\left(F_{n}\right)}{M\left(F_{n}\right)}=0$.

S'il existe une exhaustion régulière de $F^{*}$, on dira que $F^{*}$ est régulièrement exhaustible. Comme une conséquence immédiate de (1) et de (2), nous avons le

Lemme 6. Si $\left\{F_{n}\right\}$ est une exhaustion régulière de $F^{*}$ on a alors:

$$
\begin{aligned}
& e(R) \leqq \lim _{n \rightarrow \infty} \frac{e^{+}\left(F_{n}\right)}{M\left(F_{n}\right)} ; \\
& \lim _{n \rightarrow \infty} \frac{M_{n}(\gamma)}{M\left(F_{n}\right)}=1 \quad \text { pour tout arc régulier } \gamma \text { sur } R,
\end{aligned}
$$

où $M_{n}(\gamma)$ est la moyenne de nombre de feuillets de $F_{n}$ au-desses de $\gamma$.

Soit maintenant $R$ une surface de Riemann fermée, $R_{0}=R-\cup_{v=1}^{q} \delta_{v}$ le complément sur $R$ de $q(\geqq 2)$ disques fermés $\delta_{1}, \delta_{2}, \ldots, \delta_{q}$ disjoints. Soit $D$ un ouvert non borné de $\Delta^{*}: 1<|z|<\infty$ dans le plan d'une variable complexe $z$ telle que sa frontière $\partial^{*} D=\partial D \cap \Delta^{*}$ dans $\Delta^{*}$ soit composée de courbes analytiques réelles non-singulières et disjointes les unes les autres, et supposons qu'il existe une application holomorphe $\varphi$ d'un voisinage de l'adhérence $\bar{D}$ (dans le plan $z$ ) de $D$ dans $R$ vérifiant les conditions suivantes:
(i) $\varphi(D) \subset R_{0}$;
(ii) $\varphi\left(\partial^{*} D\right) \subset \partial R_{0}$;
(iii) $\varphi(\infty \mid D)=\bar{R}_{0}$,

où $\varphi(\infty \mid D)=\bigcap_{r>1} \overline{\varphi(D \cap\{|z|>r\}}$. Soit $(\gamma)_{v}$ l'ensemble des composantes simplement connexes compactes $\gamma$ de $\Delta^{*}-D$ telles que $\varphi(\partial \gamma)=\partial \delta_{v}$. Posons

$$
m_{v}=\sup \left\{\begin{array}{l|l}
m \in N & \begin{array}{l}
\text { le nombre des } \gamma \in(\gamma)_{v} \text { vérifiant } \\
|\partial \gamma| /\left|\partial \delta_{v}\right|<m \text { soit fini }
\end{array}
\end{array}\right\},
$$

où $\boldsymbol{N}$ est l'ensemble des nombres entiers positifs.

(17) 《infinite bordered covering surface》 dans [25]. 
On a alors le

Lemme 7. $\sum_{v=1}^{q}\left(1-\frac{1}{m_{v}}\right) \leqq-e(R)$.

$1^{\circ}$ En effet, nous regardons $\bar{D}$ comme une surface de recouvrement à bords de $\bar{R}_{0}$ par rapport à la projection $\varphi$, et munissons $\bar{R}_{0}$ d'une métrique conforme $d s$ à coefficient strictement positif (par exemple, la restriction sur $\bar{R}_{0}$ de la métrique de Poincaré de $R-\bigcup_{v=1}^{q} \delta_{v}^{\prime}$, chaque $\delta_{v}^{\prime}$ étant un disque fermé à l'intérieur de $\left.\delta_{v}\right)$. $\quad \bar{D}$ est alors régulièrement exhaustible. En effet, considérons $D(r)=D \cap\{1 \leqq|z| \leqq r\}$ et $C(r)=\bar{D} \cap\{|z|=r\}$ pour $r>1$. L'aire $A(r)$ de $D(r)$ et la longueur $L(r)$ de $C(r)$ serons mesurées par la métrique $\varphi^{*} d s=\mu(z)|d z|$ induite de $d s$ par $\varphi$. La longueur de $C(r)$ mesurée par la métrique euclidienne $|d z|$ sera notée $l(r)$. On déduit alors, à l'aide du lemme de Schwarz,

$$
L(r)^{2}=\left(\int_{C(r)} \mu(z)|d z|\right)^{2} \leqq l(r) \cdot \int_{C(r)} \mu(z)^{2}|d z| \leqq 2 \pi r \cdot \frac{d A(r)}{d r}
$$

d'où

$$
\frac{d r}{r} \leqq 2 \pi \cdot \frac{d A(r)}{L(r)^{2}}
$$

S'il existe un $\varepsilon>0$ tel que $L(r)>\varepsilon$ pour tout $r \geqq r_{0}$, on aura

$$
\frac{2 \pi}{\varepsilon^{2}}\left[A(r)-A\left(r_{0}\right)\right]>\int_{r_{0}}^{r} \frac{d r}{r}=\log r-\log r_{0} \rightarrow \infty \quad(r \rightarrow \infty) .
$$

Par suite, si $A(r)$ était borné, il existerait une suite de nombres positifs $1<r_{1}$ $<r_{2}<\cdots<r_{n}<\cdots$ telle que $\lim _{n \rightarrow \infty} r_{n}=\infty$ et $\lim _{n \rightarrow \infty} L\left(r_{n}\right)=0$. En remplaçant $\left\{r_{n}\right\}$ en cas de nécessité par une de ses suites partielles, on pourrait supposer que l'image $\varphi\left(C\left(r_{n}\right)\right)$ converge à un point $P$ de $\bar{R}_{0}$. Posons $G(n)=D \cap\left\{r_{n}<|z|<r_{n+1}\right\}$. Il existerait alors, quelque soit $U$ un voisinage de $\partial R_{0} \cup\{P\}$, un entier $N>0$ tel que l'on ait $\varphi(\partial G(n)) \subset U$ pour tout $n \geqq N$. Or, $\lim _{n \rightarrow \infty}|G(n)|=0, A(r)$ étant borné. Donc, si $\left|R_{0}-U\right|>0$, il existerait un entier $N^{\prime}(\geqq N)$ tel que $\varphi(G(n)) \subset U$ pour tout $n \geqq N^{\prime}$, de sorte que $\varphi\left(D \cap\left\{|z| \geqq N^{\prime}\right\}\right) \subset U$. En faisant $U \rightarrow \partial R_{0} \cup\{P\}$, on obtiendrait $\varphi(\infty \mid D) \subset \partial R_{0} \cup\{P\}$, ce qui est contraire à (iii). On a donc

$$
\lim _{r \rightarrow \infty} A(r)=\infty \text {. }
$$

Or, on a, d'après (6),

$$
2 \pi \int_{1}^{r} \frac{d A(r)}{L(r)^{2}} \geqq \int_{1}^{r} \frac{d r}{r}=\log r \rightarrow \infty, \quad \text { si } r \rightarrow \infty .
$$


Supposons qu'il existe un $\varepsilon>0$ et $r_{0}>1$ tels que l'on ait $L(r)>\varepsilon A(r)$ pour tout $r \geqq r_{0}$; on aurait alors

$$
\int_{r_{0}}^{r} \frac{d A(r)}{L(r)^{2}} \leqq \frac{1}{\varepsilon^{2}} \int_{r_{0}}^{r} \frac{d A(r)}{A(r)^{2}}=\frac{1}{\varepsilon^{2}}\left[\frac{1}{A\left(r_{0}\right)}-\frac{1}{A(r)}\right]<\frac{1}{\varepsilon^{2} A\left(r_{0}\right)},
$$

ce qui est contraire à (8). Il existe donc, une suite $r_{1}<r_{2}<\cdots<r_{n}<\cdots \rightarrow \infty$ telle que l'on ait, tenant compte de (7),

$$
\lim _{n \rightarrow \infty} \frac{L\left(r_{n}\right)+L(1)}{A\left(r_{n}\right)}=0 .
$$

On obtient ainsi une exhaustion régulière $\left\{\overline{D\left(r_{n}\right)}\right\}_{n=1,2, \ldots,}$, de $\bar{D}$.

$2^{\circ}$ Posons maintenant $D_{n}=D\left(r_{n}\right), \Delta\left(1, r_{n}\right)=\left\{z \in C|1<| z \mid<r_{n}\right\}$ et soit $D_{n}^{\prime}$ la réunion de $D_{n}$ et des composantes compactes de $\Delta\left(1, r_{n}\right)-D_{n}$. On a alors

$$
0 \geqq e\left(D_{n}^{\prime}\right)=e\left(D_{n}\right)+e\left(D_{n}^{\prime}-D_{n}\right),
$$

$D_{n}^{\prime} \cap\left(\partial D_{n}\right)$ se décomposant en des courbes simples fermées. Si l'on désigne le nombre des composantes simplement connexes $\gamma$ de $D_{n}^{\prime}-D_{n}$ tels que $\varphi(\partial \gamma)=\partial \delta_{v}$ par $N_{v, n}$, on en déduit

$$
e\left(D_{n}\right) \leqq-e\left(D_{n}^{\prime}-D_{n}\right) \leqq \sum_{v=1}^{q} N_{v, n}
$$

et, tenant compte de (4) et de (7),

$$
\varlimsup_{n \rightarrow \infty} \frac{e^{+}\left(D_{n}\right)}{M\left(D_{n}\right)} \leqq \sum_{v=1}^{q} \varlimsup_{n \rightarrow \infty} \frac{N_{v, n}}{M\left(D_{n}\right)}=\sum_{v=1}^{q} \varlimsup_{n \rightarrow \infty} \frac{N_{v, n}}{M_{n}\left(\partial \delta_{v}\right)} \leqq \sum_{v=1}^{q} \frac{1}{m_{v}}
$$

donc, d'après (3),

$$
q+e(R)=e\left(R_{0}\right) \leqq \sum_{v=1}^{q} \frac{1}{m_{v}}, \text { d'où } \sum_{v=1}^{q}\left(1-\frac{1}{m_{v}}\right) \leqq-e(R) .
$$

C.Q.F.D.

La démonstration du Théorème 4 est maintenant immédiate. En effet, rappelons les notations $S, f: \Delta^{*} \rightarrow S, C=f(0 ; S) ; \widetilde{C}_{0} \stackrel{\pi}{\longrightarrow} C_{0}, l(\widetilde{P})$ etc. du $\mathrm{n}^{\circ} 4$. Prenons un nombre fini de points $\widetilde{P}_{1}, \widetilde{P}_{2}, \ldots, \widetilde{P}_{q}$ de $\widetilde{C}_{0}$, comprenant tous les points de $\widetilde{C}_{0}$ qui correspondent par $\pi$ aux points singuliers de $C$ sur $C_{0}$, et, pour chaque $v(=1,2, \ldots, q)$, un dicylindre fermé $\Gamma_{v}:\left|x_{v}\right| \leqq 1,\left|y_{v}\right| \leqq 1^{(12)}$ de coordonées locales $x_{v}, y_{v}$ de $S$ au point $\pi\left(P_{v}\right)$ de façon que:

(i) $C \cap T_{v} \subset\left\{P \in \Gamma_{v} \mid x_{v}(P) \cdot y_{v}(P)=0\right\}$;

(ii) un petit voisinage de $\widetilde{P}_{v}$ s'applique sur $\left\{P \in \Gamma_{v} \mid y_{v}(P)=0\right\}$ par $\pi$;

(iii) $\Gamma_{\mu} \cap \Gamma_{v}=\varnothing$ pour $\pi\left(P_{\mu}\right) \neq \pi\left(P_{v}\right)$; 
(iv) $\left(\Gamma_{\mu}, x_{\mu}, y_{\mu}\right)=\left(\Gamma_{v}, y_{v}, x_{v}\right)$ pour $\pi\left(P_{\mu}\right)=\pi\left(P_{v}\right), \mu \neq v$.

Posons $\Gamma_{v}(s)=\left\{P \in \Gamma_{v}|| x_{v}(P)|<s,| y_{v}(P) \mid<s\right\}, R(s)=C_{0}-\bigcup_{v=1}^{q} \Gamma_{v}(s)$ pour chaque $0<s<1$. Prenant un $0<s_{0}<1$, soit $T$ un tube le long de $R_{0}^{v=1}=R\left(s_{0}\right)$ muni d'une rétraction analytique $\varphi: T \rightarrow R_{0}$ (cf. le Lemme 1) tel que:

(v) $T \cap C=R_{0}$;

(vi) $\Gamma_{v} \cap T \subset\left\{P \in \Gamma_{v}\left|s_{1}<\right| x_{v}(P)|\leqq 1,| y_{v}(P) \mid \leqq s_{1}\right\} \cup\left\{P \in \Gamma_{v}|| x_{v}(P) \mid<s_{1}, s_{1}\right.$ $\left.<\left|y_{v}(P)\right|<1\right\}$ pour un $0<s_{1}<s_{0}$;

(vii) $\varphi^{-1}\left(\partial R_{0}\right) \subset \cup_{v=1}^{q} \Gamma_{v}\left(s^{\prime}\right)$ pour un $s_{0}<s^{\prime}<1$.

Comme $f(0 ; S)=C$, on peut trouver un nombre réel $\rho>0$ tel que $f\left(\bar{\Delta}_{\rho}^{*}\right) \cap b T$ $=\varnothing$, où $\bar{\Delta}_{\rho}^{*}: 0<|z| \leqq \rho$ et $b T=\bigcup_{w \in R_{0}} \partial \varphi^{-1}(w)$ (cf. le Lemme 1). On peut regarder $F_{0}=\bar{\Delta}_{\rho}^{*} \cap f^{-1}(T)$ comme une surface de recouvrement à bords de $R_{0}$ par rapport à la projection $\psi=\varphi \circ f$. Choisissons $s$ dans $s_{0}<s<1$ de façon que $\varphi^{-1}(\partial R(s))$ $\subset \bigcup_{v=1}^{q} \Gamma_{v}$ et que $F_{0}$ ne ramifie pas au-dessus de $\partial R(s)$. Soit $F=\psi^{-1}(R(s))$, et soit $D$ l'ensemble des points intérieurs de $F$. Alors, $\partial^{*} D=\partial D \cap \Delta_{\rho}^{*}$ est nonsingulière (où $\left.\Delta_{\rho}^{*}: 0<|z|<\rho\right), \psi(D) \subset R(s)-\partial R(s), \psi\left(\partial^{*} D\right) \subset \partial R(s)$ et $\psi(0 \mid D)$ $=R(s)$. Soit $\delta_{v}$ la composante connexe de $\pi^{-1}\left(C_{0} \cap \Gamma_{v}(s)\right)$ contenant $\widetilde{P}_{v},(\gamma)_{v}$ l'ensemble des composantes simplement connexes compactes $\gamma$ de $\Delta_{\rho}^{*}-D$ telles que $\psi(\partial \gamma)=\pi\left(\partial \delta_{v}\right)$, et enfin

$$
m_{v}=\sup \left\{\begin{array}{l|l}
m \in \mathbb{N} & \begin{array}{l}
\text { le nombre des } \gamma \in(\gamma)_{v} \text { vérifiant } \\
\frac{1}{2 \pi i} \int_{\partial \gamma} d \log x_{v}(f(z))<m \text { soit fini }
\end{array}
\end{array}\right\} .
$$

Alors, d'après la Proposition 5 , on a $l\left(\widetilde{P}_{v}\right) \leqq m_{v}$. D'autre part, d'après le Lemme 7 , on a

$$
\sum_{v=1}^{q}\left(1-\frac{1}{m_{v}}\right) \leqq-e\left(\tilde{C}_{0}\right)
$$

d'où

$$
\sum_{v=1}^{q}\left(1-\frac{1}{l\left(\widetilde{P}_{v}\right)}\right) \leqq-e\left(\widetilde{C}_{0}\right)
$$

La suite $\left\{P_{v}\right\}_{v=1,2, \ldots, q}$ étant arbitraire (dans la mesure qu'elle contient tous les points de $\widetilde{C}_{0}$ correspondant aux points singuliers de $C$ sur $C_{0}$ ), on en déduit aussitôt que le nombre des points $\widetilde{P}$ de $\widetilde{C}_{0}$ tels que $l(\widetilde{P}) \geqq 2$ ne dépasse pas $-2 e\left(\tilde{C}_{0}\right)(<\infty)$, et que

$$
\sum_{\tilde{P} \in \widetilde{C}_{0}}\left(1-\frac{1}{l(\widetilde{P})}\right) \leqq-e\left(\widetilde{C}_{0}\right)(=2-2 g)
$$

C.Q.F.D. 
7. Liste des types de $f(0)$ compacts compris dans une courbe de valeurs exceptionnelles. Soit $S$ une surface complexe non-singulière, $E$ une courbe analytique connexe sur $S$ n'ayant que des points doubles ordinaires comme points singuliers et minimale au sens suivant: toute composante irréductible compacte exceptionelle de première espèce ${ }^{(7)}$ de $E$ passe par au moins trois points singuliers de $E$. Soit $f: \Delta^{*} \rightarrow S-E$ une application holomorphe du disque pointé $\Delta^{*}: 0<|z|<1$ dans $S-E$ telle que

$$
C=f(0 ; S)
$$

soit un sous-ensemble compact de $E$ contenant au moins deux points. $(f(0$; $S-E)=\varnothing$ ). Alors, d'après la Proposition 3,C est une courbe analytique compacte composée de composantes irréductibles de $E$. En appliquant le Théorème 4 à chaque composante irréductible de $C$, on déduit aussitôt que:

Théorème 5. (i) $C$ doit être de l'un des types $(\alpha)$ à $(\varepsilon)$ donnés dans le Tableau 1 ci-dessous ${ }^{(18)}$; (ii) Si $E_{*}=E-C \neq \emptyset, C$ est alors de type $(\gamma),\left(\gamma^{\prime}\right)$ ou $(\varepsilon)$, les lieux d'intersection de $\widetilde{E}_{*}$ avec $C$ étant indiqués dans le Tableau 2 ci-dessous ${ }^{(18)}$.

Tableau 1.

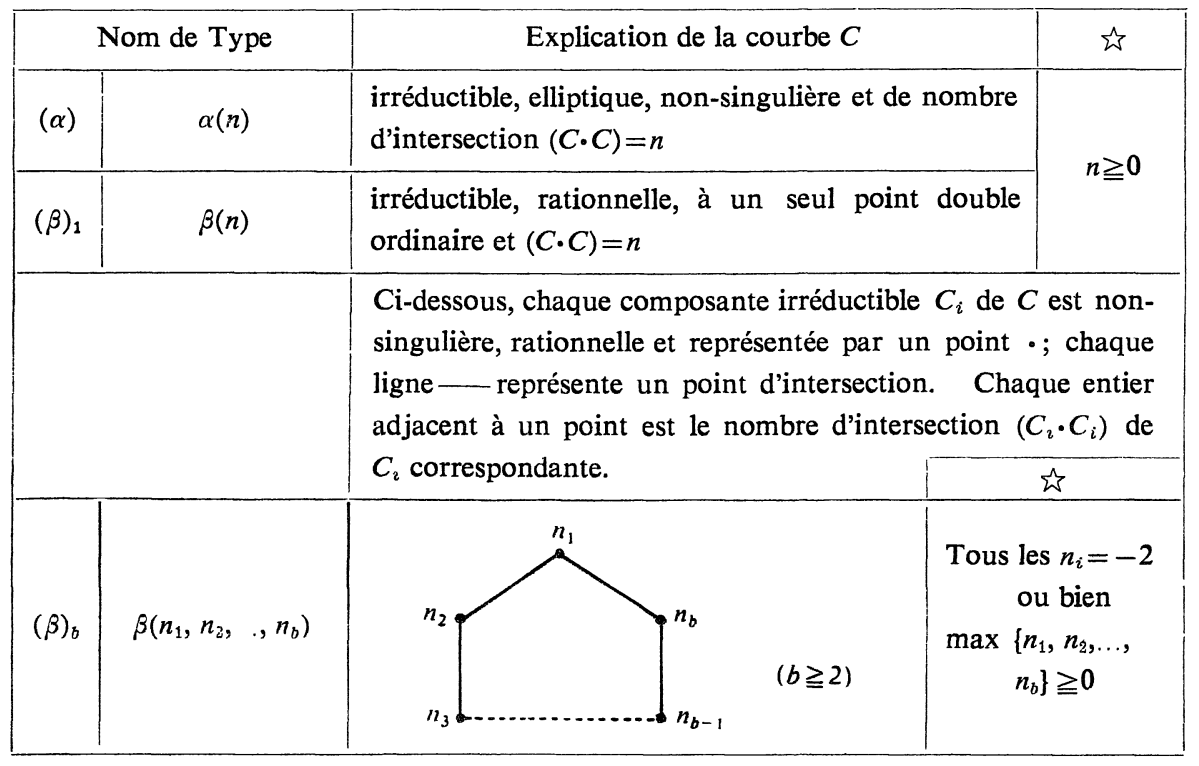

(18) Ce n'est qu'une condition nécessaire. Nous ne savons pas encore s'il existe une application holomorphe $f: \Delta^{*} \rightarrow S-C$ vérifiant $C=f(0 ; S)$ pour toutes les courbes $C$ appartenant au Tableau 1. 


\begin{tabular}{|c|c|c|c|}
\hline$(\gamma)$ & $\gamma\left(n_{1}, n_{2}, \ldots, n_{b}\right)$ & $n_{-2}^{-2}(b \geqq 1)$ & $\begin{array}{l}\text { Tous les } n_{i}=-2 \\
\quad \text { ou bien } \\
\max \left\{n_{1}+1, n_{2}, \ldots,\right. \\
\left.n_{b-1}, n_{b}+1\right\} \geqq 0\end{array}$ \\
\hline$\left(\gamma^{\prime}\right)$ & $\gamma^{\prime}\left(n_{1}, n_{2}, \ldots, n_{b}\right)$ & $\int_{-2}^{n_{1} \quad n_{2}} n_{0}^{-2}$ & $\begin{array}{cl}\max & \left\{n_{1}+1, n_{2}, \ldots,\right. \\
& \left.n_{b}\right\} \geqq 0\end{array}$ \\
\hline$(\delta)$ & $\delta\left(n_{0} \mid \frac{q_{1}}{l_{1}}, \frac{q_{2}}{l_{2}}, \frac{q_{3}}{l_{3}}\right)$ & $\begin{array}{l}\text { (1) Pour chaque } i=1,2,3,\left(l_{i}, q_{i}\right) \text { est une } \\
\text { paire d'entiers } 0<q_{i}<l_{i} \text { premiers entre } \\
\text { eux et } \\
\frac{l_{i}}{q_{i}}=n_{i, 1}-\frac{n_{2, r_{2}}}{n_{2,2}-n_{2,1}}-\frac{1}{n_{1, r_{1}}} \\
\qquad \text { où } \quad n_{i j} \geqq 2 ; \\
\text { (2) } \sum_{i=1}^{3}\left(1-\frac{1}{l_{i}}\right) \leqq 2 .\end{array}$ & $\begin{array}{l}n_{0} \geqq 2 \text {, où on a } \\
\text { l'égalité seulement } \\
\text { si: } \\
\left(\frac{q_{1}}{l_{1}}, \frac{q_{2}}{l_{2}}, \frac{q_{3}}{l_{3}}\right) \\
=\left\{\begin{array}{l}\left(\frac{1}{2}, \frac{2}{3}, \frac{5}{6}\right), \\
\left(\frac{1}{2}, \frac{3}{4}, \frac{3}{4}\right) \\
\text { ou bien } \\
\left(\frac{2}{3}, \frac{2}{3}, \frac{2}{3}\right) .\end{array}\right.\end{array}$ \\
\hline$(\varepsilon)$ & $\varepsilon\left(n_{1}, n_{2}, \cdots, n_{b}\right)$ & $n_{1} \quad n_{2} \quad n_{b}$ & $\begin{array}{c}\max \left\{n_{1}, n_{2}, \ldots,\right. \\
\left.n_{\iota}\right\} \geqq 0\end{array}$ \\
\hline
\end{tabular}

Remarque 1. Dans la colonne $\iota^{\jmath}$ du Tableau 1, on a écrit les conditions pour que la matrice des nombres d'intersections $\left(\left(C_{i} \cdot C_{j}\right)\right)$ ne soit pas négative définite. En effet, si $\left(\left(C_{i} \cdot C_{j}\right)\right)$ était négative définite, $C$ serait, d'après $\mathrm{H}$. Grauert [5], exceptionnelle; par suite, d'après la Proposition $1, f$ serait holomorphe en $z=0$, contrairement à $C=f(0 ; S)$.

Remarque 2. Pour le type $(\delta)$, il suit de la condition (2) que $\left(l_{1}, l_{2}, l_{3}\right)$ $=(2,3,6-m)$ avec $m=0,1,2,3 ;(2,4,4)$ ou $(3,3,3)$. 
Tableau 2.

\begin{tabular}{|c|c|c|c|}
\hline Nom de Type & $\begin{array}{l}\text { Nombre des } \\
\text { points de } C \cap \bar{E}_{*}\end{array}$ & Lieux de $C \cap \bar{E}_{*}$ \\
\hline$r^{\prime}\left(n_{1}, n_{2}, \ldots, n_{b}\right)$ & 1 & 1 & \\
\hline$\varepsilon\left(n_{1}, n_{2}, \ldots, n_{b}\right)$ & 2 & & \\
& & &
\end{tabular}

Corollaire 1. Soient $(S, E)$ les mêmes qu'au début du présent numéro, et $f_{v}: \Delta^{*} \rightarrow S-E(v=1,2, \ldots, n)$ un nombre fini d'applications holomorphes de $\Delta^{*}$ dans $S-E$ telles que $C^{(v)}=f_{v}(0 ; S)$ soit une courbe compacte sur $E$ et que la réunion $C=\bigcup_{v=1}^{n} C^{(v)}$ soit connexe. Alors, $C$ est aussi de l'un des types $(\alpha)$ $\grave{a}(\varepsilon) d u$ Tableau 1. En outre, si $E^{*}=E-C \neq \varnothing$, elle doit appartenir au Tableau 2.

Compléments au Tableau 1. - Supposons que $S$ est compacte. En vertu du théorème de Kodaira-Spencer [13] et du Théorème 5.1 de Kodaira [11], si $S$ ou l'une des équivalentes birationnelles de $S$ contient une courbe rationnelle non-singulière $\Theta$ telle que $(\Theta \cdot \Theta) \geqq 0, S$ est une surface réglée (ruled surface). Donc, $S$ est une surface réglée, si $C$ est de l'un des types suivants: $\beta(n)$ avec $n \geqq 4 ; \beta\left(n_{1}, n_{2}, \ldots, n_{b}\right)$ avec $b \geqq 2, \max \left\{n_{1}, n_{2}, \ldots, n_{b}\right\} \geqq 0 ; \gamma\left(n_{1}, n_{2}, \ldots, n_{b}\right)$ avec $\max \left\{n_{1}+1, n_{2}, \ldots, n_{b-1}, n_{b}+1\right\} \geqq 0 ; \gamma^{\prime}\left(n_{1}, n_{2}, \ldots, n_{b}\right) ; \delta\left(n_{0} \mid \frac{q_{1}}{l_{1}}, \frac{q_{2}}{l_{2}}, \frac{q_{3}}{l_{3}}\right)$ avec $n_{0} \geqq 0 ; \delta\left(-1 \mid \frac{q_{1}}{l_{1}}, \frac{q_{2}}{l_{2}}, \frac{q_{3}}{l_{3}}\right)$ avec $n_{i, 1}=2$ pour au moins $\operatorname{deux} i \in\{1,2,3\}$; $\delta\left(-1 \mid \frac{1}{3}, \frac{1}{3},-\frac{2}{3}\right)$ et $\varepsilon\left(n_{1}, n_{2}, \ldots, n_{b}\right)$. D'autre part, d'après le Théorème 56 de Kodaira [12], si une surface complexe non-singulière et compacte contient deux courbes exceptionnelles de première espèce qui intersectent l'une l'autre, elle est réglée. Donc, $S$ est réglée pour les cas suivants également: $\beta(3)$, $\delta\left(-1 \mid \frac{1}{2}, \frac{1}{3}, \frac{1}{3}\right)$ et $\delta\left(-1 \mid \frac{1}{2}, \frac{1}{3}, \frac{2}{5}\right)$. Considérons enfin une surface 
complexe non-singulière et compacte $S_{1}$ contenant une courbe analytique irréductible $C_{1}$ avec $\left(C_{1} \cdot C_{1}\right)=n>0$ et $\pi\left(C_{1}\right)$ (le genre virtuel de $\left.C_{1}\right)=1$; alors, d'après K. Kodaira [11] (Th. 3.3), $S$ est algébrique; d'autre part, $K_{1}$ étant le diviseur canonique de $S_{1}, \pi\left(C_{1}\right)=\frac{\left(K_{1} \cdot C_{1}\right)+\left(C_{1}^{2}\right)}{2}+1=1$ entraine $\left(K_{1} \cdot C_{1}\right)<0$, de sorte que $P_{m}=\operatorname{dim} H^{0}\left(S_{1}, m K_{1}\right)=0$ pour tout $m>0$; donc, d'après $\mathrm{F}$. Enriques (cf. I. R. Šafarevič [23], Chap. IV), $S$ est une surface réglée. Par conséquent, si $C$ est de l'un des types $\alpha(n), \beta(n), \delta\left(-1 \mid \frac{1}{2}, \frac{1}{3}, \frac{1}{6-n}\right)$ avec $n>0, S$ est réglée. ${ }^{*}$ Nous obtenons ainsi le

Corollaire 2. Dans la situation $d u$ Théorème 1 , si $S$ est une surface compacte non-réglée, alors $C$ est de l'un des dix types suivants (Tableau 3):

Tableau 3.
(1) $\alpha(0)$;
(2) $\beta(0)$;

(3) $\beta(-2,-2, \ldots,-2)=$

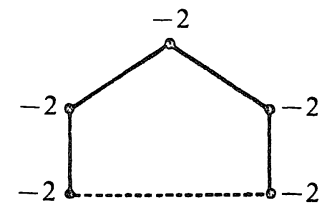

(le nombre des points $\geqq 2$ );

(4) $r(-2,-2, \ldots,-2)=$



( 5) $\delta\left(-1 \mid \frac{1}{2}, \frac{1}{3}, \frac{1}{6}\right)=$

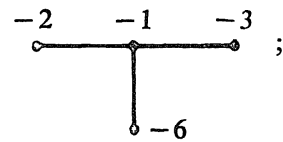

(6) $\delta\left(-1 \mid \frac{1}{2}, \frac{1}{4}, \frac{1}{4}\right)=$



(7) $\delta\left(-1 \mid \frac{1}{3}, \frac{1}{3}, \frac{1}{3}\right)=$

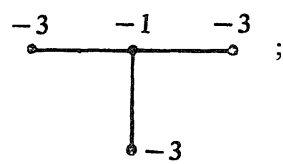

(8) $\delta\left(-2 \mid \frac{1}{2}, \frac{2}{3}, \frac{5}{6}\right)=$



(*) Nous devons cet imposibilité de $\alpha(n), \beta(n), \delta\left(-1 \mid \frac{1}{2}, \frac{1}{3}, \frac{1}{6-n}\right)$ avec $n>0$ dans le Tableau 3 ci-dessous au Referee du présent article, que nous voudrions remercier ici. 
(9)

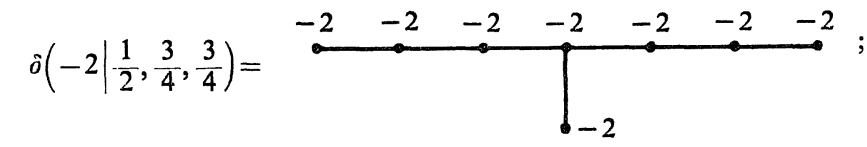

(10) $\delta\left(-2 \mid \frac{2}{3}, \begin{array}{ll}2 & 2 \\ 3 & 3\end{array}\right)=$

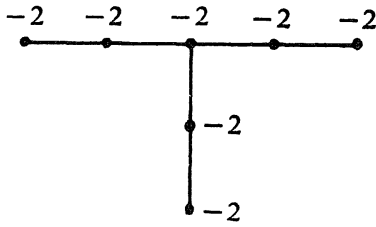

Remarque. - Si $S$ est une surface compacte réglée non-rationnelle (c'està-dire qu'il existe une application holomorphe propre $\pi: S \rightarrow R$ de $S$ sur une courbe compacte $R$ de genre $\geqq 1$ dont les fibres génériques $\pi^{-1}(t)$ sont isomorphes à la droite projective $\left.\mathbb{P}^{1}\right)$, alors $C=f(0 ; S)$ est ou bien un fibre de $\pi$, ou bien $R$ est de genre 1 et $C$ de type $\alpha(n)$.

\section{Chapitre III. - Singularités Essentielles des Isomorphismes Analytiques le Long d'une Courbe Compacte}

8. $\varphi_{\text {ess }}(\Sigma)$. Soient $S, S^{\prime}$ deux surfaces complexes non-singulières et $E, E^{\prime}$ deux courbes analytiques compactes et connexes sur $S, S^{\prime}$ respectivement. Soit $\varphi: S-E \rightarrow S^{\prime}-E^{\prime}$ une application holomorphe non-dégénérée. Pour chaque sous-ensemble fermé $K$ de $E$, nous poserons

$$
\varphi\left(K ; S^{\prime}\right)=\underset{U \in \mathfrak{u}(K)}{\bigcap(U-E)},
$$

où $\mathfrak{U}(K)$ est un système fondamental de voisinages de $K$ et $\overline{\varphi(U-E)}$ l'adhérence dans $S^{\prime}$ de $\varphi(U-E)$. Nous supposerons dans ce qui suit que:
(1) $\varphi\left(E ; S^{\prime}\right) \subset E^{\prime} \quad$ et
(2) $\varphi\left(E ; S^{\prime}\right) \neq$ vide.

Comme on le vérifie aisément, il existe une paire de voisinages $V(\Subset S)$, $V^{\prime}\left(\Subset S^{\prime}\right)$ de $E, E^{\prime}$ respectivement tels que $\varphi_{\mid V-E}: V-E \rightarrow V^{\prime}-E^{\prime}$ soit propre et surjective. On a, par conséquent, $\varphi\left(E ; S^{\prime}\right)=E^{\prime}$.

On dira que $\varphi$ est méromorphe à un point $P$ de $E$, s'il existe un voisinage $U=U(P)$ de $P$ tel que l'adhérence $\overline{G_{U}}$ dans $U \times S^{\prime}$ du graphique

$$
G_{U}=\{(x, \varphi(x)) \mid x \in U-E\}
$$

soit un sous-ensemble analytique de $U \times S^{\prime}$. Au cas contraire, on dira que $P$ 
est un point singulier essentiel de $\varphi$ (par rapport à $S^{\prime}$ ). L'ensemble de ces points singuliers essentiels de $\varphi$ sur $E$ sera désigné par $\Sigma$.

D'après l'hypothèse (1), le graphique $G=\{(x, \varphi(x)) \mid x \in S-E\}$ de $\varphi$ est un sous-ensemble analytique de $S \times S^{\prime}-E \times E^{\prime}$ de dimension pure 2. Comme $E \times E^{\prime}$ est un sous-ensemble analytique de $S \times S^{\prime}$ de dimension pure 2 , il en résulte que, d'après le théorème de Remmert-Stein cité au no 3 , $1^{\prime}$ 'ensemble $\mathfrak{U}$ des points $(x, y)$ de $E \times E^{\prime}$ tels que l'adhérence $\bar{G}$ de $G$ dans $S \times S^{\prime}$ ne soit pas analytique $^{(19)}$ en $(x, y)$ est une réunion (finie) de composantes irréductibles de $E \times E^{\prime} . \quad$ Par définition, la projection sur $S$ de $\mathfrak{A}$ coïncide avec $\Sigma$. Nous désignerons par $\Sigma^{\prime}=\varphi_{\text {ess }}(\Sigma)$ la projection sur $S^{\prime}$ de $\mathfrak{A}$ (et l'appellerons l'ensemble limite essentiel de $\varphi$ dans $S^{\prime}$ le long de $\Sigma$ ).

Soient $\left\{E_{i}\right\}_{i \in I}$ (resp. $\left\{E_{j}^{\prime}\right\}_{j \in J}$ ) les composnantes irréductibles de $E$ (resp. de $\left.E^{\prime}\right)$. Alors, il est clair que les composantes irréductibles de $E \times E^{\prime}$ sont $\left\{E_{i}\right.$ $\left.\times E_{j}^{\prime}\right\}_{(i, j) \in I \times J}$. On a donc, la

Proposition 6. $\Sigma\left(\right.$ resp. $\left.\varphi_{\text {ess }}(\Sigma)\right)$ est une courbe analytique compacte dans $S$ (resp. $\left.S^{\prime}\right)$ composée de composantes irréductibles de $E$ (resp. de $E^{\prime}$ ).

Pour chaque composante irréductible $\Sigma_{i}\left(i \in I_{0} \subset I\right)$ de $\Sigma$, nous désignerons la réunion $\underset{\Sigma_{i} \times E_{j}^{\prime} \subset \mathfrak{Q}}{\cup} E_{j}^{\prime}\left(\subset \Sigma^{\prime}\right) \operatorname{par} \varphi_{\text {ess }}\left(\Sigma_{i}\right)$. On a

(3) $\Sigma^{\prime}=\varphi_{\text {ess }}(\Sigma)=\bigcup_{i \in I_{0}} \varphi_{\text {ess }}\left(\Sigma_{i}\right)$.

Lemme 8. Soit $i \in I_{0} ; \Gamma:|z|<1,|w|<1$ un voisinage de coordonnées locales $z, w$ de $S$ en un point régulier a de $\Sigma_{i}$ tel que $\Gamma \cap E=\{P \in \Gamma \mid z(P)=0\}$ $\left(\subset \Sigma_{i}\right)$. Considérons les applications holomorphes $f_{i}: \Delta^{*} \rightarrow S^{\prime}$ définies par

$$
f_{i}(z)=\varphi(z, w), \quad(0<|z|<1 ; w: \text { fixe dans }|w|<1),
$$

en identifiant $(z, w)$ avec le point de $\Gamma$ de coordonnées $z$, w. Alors, pour presque tout ${ }^{(20)} w$ dans $|w|<1$, on a

$$
f_{i}\left(0 ; S^{\prime}\right)=\varphi_{\text {ess }}\left(\Sigma_{i}\right) \text {. }
$$

En effet, prenons un point régulier $b_{j}$ de $E^{\prime}$ sur chaque composante irréductible $\Sigma_{j}^{\prime}$ de $\varphi_{\text {ess }}\left(\Sigma_{i}\right)$, et un voisinage $\Gamma_{j}^{\prime}:\left|z_{j}\right|<2,\left|w_{j}\right|<2$ de coordonnées locales $z_{j}, w_{j}$ de $S^{\prime}$ en $b_{j}$ de façon que $\Gamma_{j}^{\prime} \cap E^{\prime}=\left\{Q \in \Gamma_{j}^{\prime} \mid z_{j}(Q)=0\right\}$. Posons

(19) On dira qu'un fermé $\bar{G}$ d'une variété analytique complexe $M$ est analytique en un point $P \in M$, s'il existe un voisinage $U=U(P)$ de $P$ tel que $\bar{G} \cap U$ soit un sous-ensemble analytique de $U$.

(20) Le sens du mot 《presque tout》 sera clarifié dans la démonstration. 
$\Gamma_{j}=\left\{Q \in \Gamma_{j}^{\prime}|| z_{j}(Q)|<1,| w_{j}(Q) \mid<1\right\}$. Considérons dans le disque unité $\Delta_{(w)}$ : $|w|<1$ du plan $w$ l'ensemble

$$
K_{j}(n)=\left\{w \in \Delta_{(w)} \mid \varphi(z, w) \notin \Gamma_{j} \quad \text { pour } \quad 0<|z| \leqq \frac{1}{n}\right\}
$$

pour chaque entier $n \geqq 2$. Ce sont fermés dans $\Delta_{(w)}$. Nous allons montrer que la réunion $K_{j}=\bigcup_{n=2}^{\infty} K_{j}(n)$ est de capacité logarithmique nulle. En effet, si au contraire $\operatorname{Cap}\left(K_{j}\right)>0$, on aurait un entier $N \geqq 2$ tel que $\operatorname{Cap}\left(K_{j}(N)\right)>0$. Soit $w_{0}$ un point de $K_{j}(N)$ tel que $\operatorname{Cap}\left(K_{j}(N) \cap \varepsilon\right)>0$ pour tout voisinage $\varepsilon$ de $w_{0}$. Comme $w_{0} \in K_{j}(N)$, on a

$$
\left(\left\{P \in \Gamma|| z(P) \mid \leqq 1 / N, w(P)=w_{0}\right\} \times \Gamma_{j}\right) \cap G=\varnothing,
$$

où $G$ est le graphique de $\varphi$ dans $S \times S^{\prime}$. G étant fermé dans $S \times S^{\prime}-E \times E^{\prime}$, on peut trouver un $\varepsilon>0$ tel que

$$
\text { (5) }\left\{\begin{array}{l|l}
(P, Q) \in \Gamma \times \Gamma_{j} & \begin{array}{l}
\max \left(|z(P)|,\left|z_{j}(Q)\right|\right)=\frac{1}{N}, \\
\max \left(\left|w(P)-w_{0}\right|,\left|w_{j}(Q)\right|\right)<\varepsilon
\end{array}
\end{array}\right\} \cap G=\varnothing
$$

Posons $U=\left\{P \in \Gamma|| z(P)|<1 / N| w,(P)-w_{0} \mid<\varepsilon\right\}, \quad U_{j}=\left\{Q \in \Gamma_{j}|| z_{j}(Q) \mid<1 / N\right.$, $\left.\left|w_{j}(Q)\right|<\varepsilon\right\}$. Comme on a pris $\Sigma_{j}^{\prime} \subset \varphi_{\text {ess }}\left(\Sigma_{i}\right)$, il existe un point $\left(P_{j}, Q_{j}\right)$ de $G$ dans $U \times U_{j}$. L'image réciproque $R_{j}$ dans $U$ de $\left\{Q \in U_{j} \mid w_{j}(Q)=w_{j}\left(Q_{j}\right)\right\}$ par $\varphi_{\mid U}$ est donc, compte tenu (5), un sous-ensemble analytique de $U$ de dimension pure un (courbe analytique). D'après (5) de nouveau, on a $\bar{R}_{j} \cap\{P \in \bar{U} \mid$ $|z(P)|=1 / N\}=\varnothing ;$ donc

(6) $\left|w(P)-w_{0}\right|=0$ ou bien $z(P)=0$ pour $P \in \partial R_{j}$.

Prenons maintenant un sous-ensemble compact $\kappa$ de $K_{j}(N) \cap\left[\left|w-w_{0}\right|<\varepsilon\right]$ de capacité logarithmique non nulle, et soit $h(w)$ la solution du problème de Dirichlet

$$
h(w)= \begin{cases}0, & \left|w-w_{0}\right|=\varepsilon \\ 1, & w \in \kappa\end{cases}
$$

dans $D ; h(w)$ est une fonction harmonique et $0<h(w)<1$ dans $D-\kappa$. Or, puisque $w(x) \notin \kappa$ pour $x \in R_{j}, h(w(x))$ est une fonction harmonique bornée sur (la normalisée de) $R_{j}$. D'autre part, on a une autre fonction harmonique négative $\log |z(x)|(<-\log N)$ sur $R_{j}$, vérifiant (d'après (6)):

$$
\lim _{\substack{x \rightarrow P \\ x \in R_{j}}} h(w(x))=0 \text { pour tout } P \in \partial R_{j} \text { tel que } \log |z(P)| \neq-\infty .
$$


On a donc, d'après le théorème de Riesz, $h(w(x)) \equiv 0$ sur $R_{j}$, ce qui est absurde, puisque pour le point $P_{j} \in R_{j}$ on a $w\left(P_{j}\right) \in D$ et $h\left(w\left(P_{j}\right)\right)>0$. Donc, Cap $\left(K_{j}\right)$ $=0$.

Le nombre des composantes irréductibles $\Sigma_{j}^{\prime}$ de $\varphi_{\text {ess }}\left(\Sigma_{i}\right)$ étant fini, $K=$ $\cup K_{j}$ est aussi de capacité logarithmique nulle. Prenons un point quelconque $\boldsymbol{\Sigma}_{j}^{\prime}$

$w_{i}$ de $\Delta_{(w)}-K$, et posons $f_{i}(z)=\left(z, w_{i}\right), 0<|z|<1$. Alors $f_{i}\left(0 ; S^{\prime}\right)$ contient tous les $b_{j}$; par suite, d'après la Proposition 3 , on a $\varphi_{\text {ess }}\left(\Sigma_{i}\right) \subset f_{i}\left(0 ; S^{\prime}\right)$. L'inclusion de sens opposée étant évidente, on a donc $\varphi_{\text {ess }}\left(\Sigma_{i}\right)=f_{i}\left(0 ; S^{\prime}\right)$ C. Q. F.D.

Ce lemme nous permet d'appliquer le Corollaire 1 du Théorème 5 à $\varphi_{\text {ess }}(\Sigma)$. Nous obtenons ainsi le

Théorème 6. Soient $S, S^{\prime}$ deux surfaces complexes non-singulières; $E, E^{\prime}$ deux courbes analytiques compactes et connexes sur $S, S^{\prime}$ respectivement, n'ayant, toues les deux, que des points doubles ordinaires comme points singuliers et minimales au sens dit au début de $\mathrm{n}^{\circ} 7$, et soit $\varphi: S-E \rightarrow S^{\prime}-E^{\prime}$ une application holomorphe non-dégénérée de $S-E$ dans $S^{\prime}-E^{\prime}$ vérifiant:


rapport à $\left.S^{\prime}\right) \neq$ vide. Alors, (i) $\Sigma^{\prime}=\varphi_{\text {ess }}(\Sigma)$ est de l'un des types $(\alpha) \grave{a}(\varepsilon) d u$ Tableau $1 d u \mathrm{n}^{\circ} 7$ (poser $C=\Sigma^{\prime}$ ); (ii) si $E^{\prime} \neq \Sigma^{\prime}, \Sigma^{\prime}$ est alors de type $(\gamma),\left(\gamma^{\prime}\right)$

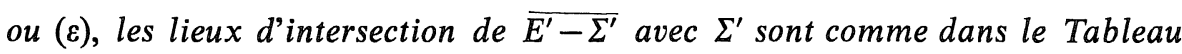
2 (n० 7 , poser $C=\Sigma^{\prime}, E_{*}=E^{\prime}-\Sigma^{\prime}$ ). (iii) Si de plus $\varphi$ est un isomorphisme analytique de $S-E$ sur $S^{\prime}-E^{\prime}$, on a les mêmes assertions que (i) et (ii) ci-dessus pour $(\Sigma, E)$ à la place de $\left(\Sigma^{\prime}, E^{\prime}\right)$. (Remarque: si $\psi$ est l'application inverse de $\varphi$, on a $\left.\Sigma=\psi_{\text {ess }}\left(\Sigma^{\prime}\right)\right)$.

9. Rapport avec la dimension de Kodaira logarithmique. Considérons le complément $V=S-C$ d'une courbe analytique compacte $C$ dans une surface complexe non-singulière et compacte $S$. S. Iitaka [8] a introduit un invariant $\bar{\kappa}(V)$ (logarithmic Kodaira dimension) de $V$ comme suit ${ }^{(21)}$ : Après un nombre fini d'éclatements successifs de points singuliers de $C$, on peut supposer que les points singuliers de $C$ sont des points doubles ordinaires (F. Hirzebruch [6]). Soient $K_{S}$ le fibré canonique en droites complexes de $S$ et $[C]$ le fibré en droites complexes défini par le diviseur $C$. On prend, pour chaque entiers $m>0$, une base $\left(\varphi_{1}, \varphi_{2}, \ldots, \varphi_{N}\right)(N=N(m))$ de l'espace vectoriel complexe $H^{\circ}\left(S, \mathcal{O}\left(m K_{S}+m[C]\right)\right)$

(21) Voir aussi F. Sakai [24]. 
des sections holomorphes du fibré $m\left(K_{S}+[C]\right)$. Considérant l'application méromorphe

$$
\Phi_{m}: S \ni P \longmapsto\left(\varphi_{1}(P), \varphi_{2}(P), \ldots, \varphi_{N}(P)\right) \in \boldsymbol{P}^{\mathrm{v}-1},
$$

on définit $\bar{\kappa}(V)$ par

$$
\bar{\kappa}(V)=\left\{\begin{array}{l}
\max \left\{\operatorname{dim} \Phi_{m}(S) \mid N(m)>0\right\}, \quad \text { s'il existe } m>0 \text { tel que } N(m)>0 \\
-\infty, \quad \text { si } N(m)=0 \text { pour tout } m>0 .
\end{array}\right.
$$

Avec S. Iitaka [8], on dira que $V$ est de type hyperbolique (ou de type général), si $\bar{\kappa}(V)=2$. (Remarque: La condition $\bar{\kappa}(V)=2$ ne dépend que de $V([8])$ ).

Dans ce qui suit, on supposera que les points singuliers de $C$ sont seulement des points doubles ordinaires et que $C$ soit minimale dans cette catégorie (cf. le début du $\left.n^{\circ} 7\right)$. Considérons une application holomorphe $f: \Delta^{*} \rightarrow V$ du disque pointé $\Delta^{*}: 0<|z|<1$ dans $V$ telle que $f(0 ; V)=\varnothing$, c'est-à-dire

$$
f(0 ; S) \subset C \text {, }
$$

et supposons que $\Sigma=f(0 ; S)$ contienne au moins deux points. $\quad \Sigma$ est alors une courbe analytique compacte et connexe composée de composantes irréductibles de $C$, et d'après le Théorème 5 , appartient au Tableau 1 ou bien au Tableau 2 suivant que $\Sigma=C(\Sigma)$ ou non, où $C(\Sigma)$ est la composante connexe de $C$ contenant $\Sigma$.

Proposition 7. Si $\Sigma \neq C(\Sigma)$, il existe alors une application holomorphe $\pi: V \rightarrow R$ de $V$ sur une courbe algébrique non-singulière et non-compacte $R$ vérifiant les deux conditions suivantes: $1^{\circ} \pi$ se prolonge en une application méromorphe $\bar{\pi}: S \rightarrow \bar{R}$ de $S$ sur le compactifié $\bar{R}$ de $R\left(\bar{\pi}_{\mid V}=\pi\right) ; 2^{\circ}$ les fibres régulières de $\pi$ sont isomorphes à $\boldsymbol{C}$ ou bien à $\boldsymbol{C}^{*}=\boldsymbol{C}-\{0\} . \quad$ (En particulier, on $a \bar{\kappa}(V) \leqq 1, c f .[8])$.

En effet, $\Sigma$ appartenant au Tableau 2, considérons d'abord le cas où $\Sigma$ est de type $\varepsilon\left(n_{1}, n_{2}, \ldots, n_{b}\right)$. Comme $\max \left\{n_{1}, n_{2}, \ldots, n_{b}\right\} \geqq 0$, on a une composante irréductible $\Sigma_{i}$ (non-singulière et rationnelle) de $\Sigma$ telle que $\left(\Sigma_{i}^{2}\right)=n_{i} \geqq 0$. Comme $C(\Sigma) \neq \Sigma$, il existe au moins un point singulier de $C$ sur $\Sigma_{i}$. En éclatant $n_{i}$ fois successivement ce point $P$, on peut supposer $\left(\Sigma_{i}^{2}\right)=0$. $S$ est donc, en vertu du théorème de Kodaira-Spencer [13] et du Théorème 5.1 de Kodaira [11], une surface algébrique et il existe une application holomorphe $\pi: S \rightarrow \bar{R}$ de $S$ sur une courbe compacte $\bar{R}$ telle que $\Sigma_{i}$ soit une fibre régulière de $\bar{\pi}$. Comme il n'y a qu'un ou deux points singuliers de $C$ sur $\Sigma_{i}$ qui sont des points 
doubles ordinaires, $F_{t}=\bar{\pi}^{-1}(t) \cap V$ sont isomorphes à $\boldsymbol{C}$ ou à $\mathbb{C}^{*}$ pour $t \in R$ voisin de $t_{0}=\bar{\pi}\left(\Sigma_{i}\right)$. En posant $R=\bar{\pi}(V), \pi=\bar{\pi}_{\mid V}: V \rightarrow R$, on obtient l'application voulue.

Il en est de même pour le cas où $\Sigma$ est de type $\gamma^{\prime}\left(n_{1}, n_{2}, \ldots, n_{b}\right)$, si max $\left\{n_{2}\right.$, $\left.n_{3}, \ldots, n_{b}\right\} \geqq 0$. Considérons donc le cas où $\left(\Sigma_{1}^{2}\right)=n_{1} \geqq-1$. En éclatant $n_{1}+1$ fois succesivement un point singulier de $C$ sur $\Sigma_{1}$, on peut faire $n_{1}=-1$, de sorte que le diagramme associé à $\Sigma$ soit:



Puis, en contractant deux composantes correspondant à la partie de ce diagramme, on obtient fication de $(S, C)$ de cette manière, on a une composante irréductible (nonsingulière et rationnelle) $\Sigma_{0}$ de $\Sigma$ telle que $\left(\Sigma_{0}^{2}\right)=0$ qui intersecte $C^{\prime}=\overline{C-\Sigma_{0}}$ en un seul point tangentiellement avec le nombre d'intersection $\left(\Sigma_{0} \cdot C^{\prime}\right)=2$. De même qu'au cas précédent, $S$ (ainsi modifiée) admet une structure de surface réglée $\bar{\pi}: S \rightarrow \bar{R}$ sur une courbe compacte $\bar{R}$ contenant $\Sigma_{0}$ comme une fibre régulière. Puisque $\left(\Sigma_{0} \cdot C^{\prime}\right)=2$, les fibres régulières de $\pi=\bar{\pi}_{\mid V}$ sont isomorphes à $\boldsymbol{C}^{*} ; \pi$ est donc l'application voulue, ce qui achève la démonstration de la Proposition 7 .

Proposition $8^{(22)}$. Si $\Sigma=C(\Sigma)$, on a $\bar{\kappa}(V) \leqq 1$.

En effet, d'après le Théorème $5, \Sigma$ appartient au Tableau 1. D'abord, si $\Sigma$ est de type $(\varepsilon),\left(\gamma^{\prime}\right), \gamma\left(n_{1}, n_{2}, \ldots, n_{b}\right)$ avec $\max \left\{n_{1}+1, n_{2}, \ldots, n_{b-1}, n_{b}+1\right\} \geqq 0$ ou $\beta\left(n_{1}, n_{2}, \ldots, n_{b}\right)$ avec $b \geqq 2$ et $\max \left\{n_{1}, n_{2}, \ldots, n_{b}\right\} \geqq 0$, il existe alors, de même qu'on l'a vu dans la Proposition 7, une application méromorphe $\pi$ de $S$ sur une courbe $R$ telle que $\pi_{\mid V}$ soit holomorphe et que les fibres génériques de $\pi_{\mid V}$ soient isomorphes à $\boldsymbol{C}$ ou bien à $\boldsymbol{C}^{*}$, de sorte que $\bar{\kappa}(V) \leqq \operatorname{dim} R=1$ (cf. [8]). Il nous reste donc qu'à examiner les cas où $\Sigma$ est de types suivants:

(i) $\alpha(n), \beta(n), \beta(-2,-2, \ldots,-2)(b \geqq 2)$;

(ii) $\gamma(-2,-2, \ldots,-2)$;

(iii) $\delta\left(n_{0} \mid \frac{q_{1}}{l_{1}}, \frac{q_{2}}{l_{2}}, \frac{q_{3}}{l_{3}}\right)$.

(22) Nous voudrions remercier ici le Referee et $M$. Ishida qui nous ont indiqué l'erreur que contenait la preuve de cette Proposition à la première rédaction du présent article. 
Supposons qu'il existe un entier $m>0$ tel que le système linéaire $\left|m\left(K_{S}+C\right)\right|$ soit de dimension $\geqq 1$. Ecrivons chaque $D_{\lambda} \in\left|m\left(K_{S}+[C]\right)\right|$ comme suit:

$$
D_{\lambda}=F_{\lambda}+\sum_{i} a_{i} \quad \text { (composante irréductible de } \Sigma \text { ) }
$$

où $a_{i}$ sont des entiers $\geqq 0$ et $F_{\lambda}$ (diviseur effectif) n'a pas de composantes communes avec $\Sigma$, et considérons le diviseur effectif $\tilde{\Sigma}$ défini comme suit:

- dans le cas (i), on pose $\widetilde{\Sigma}=\Sigma$;

— dans le cas (ii), $\tilde{\Sigma}=\sum_{i=1}^{4} E_{i}+2 \sum_{j=1}^{b} G_{j},\left\{E_{i}\right\},\left\{G_{j}\right\}$ étant les composantes irréductibles de $\Sigma$ indiquées dans le diagramme suivant de $\Sigma$ :



— dans le cas (iii), $\left\{G_{0}, G_{i j} ; i=1,2,3 ; j=1,2, \ldots, r_{i}\right\}$ étant les composantes irréductibles de $\Sigma$ qui correspondent, dans le diagramme de $\Sigma$ donné dans le Tableau $1\left(\mathrm{n}^{\circ} 7\right)$ pour le type $(\delta)$, aux points avec les poids $\mathrm{n}_{0},-n_{i j}$ respectivement (de sorte que $\left(G_{0}^{2}\right)=n_{0},\left(G_{i j}^{2}\right)=-n_{i j}$ ), on pose $\tilde{\Sigma}=m_{0} G_{0}+\sum_{i=1}^{3} \sum_{j=1}^{r i} k_{i} m_{i j} \cdot G_{i j}$, où $m_{0}$ est un multiple commun de $l_{1}, l_{2}, l_{3}, k_{i}=m_{0} / l_{i}$ et $m_{i j}$ sont les entiers $>0$ définis par l'argorithme suivant:

$$
\begin{gathered}
\text { (业) }\left\{\begin{array}{l}
m_{i, r_{i}}=1, m_{i, r_{i}-1}=n_{i, r_{i}} \cdot m_{i, r_{i}} \\
m_{i, r_{i}-2}=n_{i, r_{i}-1} \cdot m_{i, r_{i}-1}-m_{i, r_{i}} \\
\cdots \\
m_{i, 0}=n_{i, 1} \cdot m_{i, 1}-m_{i, 2} \quad\left(m_{i, 0}=l_{i}, m_{i, 1}=q_{i}\right) .
\end{array}\right. \\
\left(\text { Rappeler } \frac{l_{i}}{q_{i}}=n_{i, 1}-\frac{1}{n_{i, 2}-}\right) . \\
\quad-\frac{1}{n_{i, r_{i}}}
\end{gathered}
$$

Nous allons montrer $F_{\lambda} \cdot \tilde{\Sigma}=0$. Voyons d'abord $\left(K_{S}+\Sigma\right) \cdot \tilde{\Sigma} \leqq 0$; ce qui peut être vérifié aisément pour le cas (i) et (ii) (cf. [11], I, p. 119); pour le cas (iii), on a

$$
\begin{aligned}
\left(K_{S}+\Sigma\right) \cdot \tilde{\Sigma}= & m_{0}\left(K_{S}+G_{0}\right) G_{0}+\sum_{i, j} k_{i} m_{i j}\left(K_{S}+G_{i j}\right) G_{i j} \\
& +m_{0} \sum_{i, j} G_{i j} \cdot G_{0}+\sum_{i, j} k_{i} m_{i j}\left(G_{0} \cdot G_{i j}+\sum_{(\lambda, \mu) \neq(i, j)} G_{\lambda \mu} \cdot G_{i j}\right) \\
= & m_{0}-\sum_{i=1}^{3} k_{i}=m_{0}\left(1-\sum_{i=1}^{3} \frac{1}{l_{i}}\right) \leqq 0 .
\end{aligned}
$$


On a donc $\left(K_{S}+C\right) \cdot \tilde{\Sigma} \leqq 0$, d'où
(a)
$D_{\lambda} \cdot \tilde{\Sigma} \leqq 0$.

Or, pour toute composante irréductible $G_{*}$ de $\Sigma$, on a

(b) $\quad G_{*} \cdot \tilde{\Sigma} \geqq 0$.

En effet, il est évident pour les cas (i) et (ii); plaçons-nous donc au cas (iii): d'abord, $G_{i, j} \cdot \tilde{\Sigma}=0$ se résulte immédiatement de l'argorithme ( $\left.\tilde{\zeta}\right)$ ci-dessus; d'autre part, comme $\Sigma$ n'est pas exceptionnelle, le déterminant de la matrice $\left(-\left(\Sigma_{\lambda} \cdot \Sigma_{\mu}\right)\right),\left\{\Sigma_{\lambda}\right\}$ étant les composantes irréductibles de $\Sigma$, égale à

$$
-a \cdot\left(n_{0}+\sum_{i=1}^{3} \frac{q_{i}}{l_{i}}\right), \text { avec } a>0,
$$

est $\leqq 0$; on a donc,

$$
G_{0} \cdot \tilde{\Sigma}=m_{0} n_{0}+\sum_{i=j}^{3} k_{i} q_{i}=m_{0}\left(n_{0}+\sum_{i=1}^{3} \frac{q_{i}}{l_{i}}\right) \geqq 0 .
$$

D'après (a) et (b), on obtient $F_{\lambda} \cdot \tilde{\Sigma} \leqq 0$ (de sorte que $F_{\lambda} \cdot \tilde{\Sigma}=0$ ).

Maintenant, d'après l'hypothèse $\operatorname{dim}\left|m\left(K_{S}+[C]\right)\right| \geqq 1$, il existe au moins deux sections holomorphes $\varphi_{0}, \varphi_{1}$, linéairement indépendantes, de $m\left(K_{S}+[C]\right)$ sur $S$, qui donnent une fonction méromorphe

$$
\Phi: \quad S \ni P \longmapsto\left(\varphi_{0}(P), \varphi_{1}(P)\right) \in \mathbb{P}^{1}
$$

non-constante sur $S$. Alors, pour presque tout $t \in \mathbb{P}^{1}$, la fibre $\Phi^{-1}(t)$ est composée de composantes du diviseur $F_{\lambda}$ associé à $D_{\lambda} \in\left|m\left(K_{S}+[C]\right)\right|$ défini par $\lambda_{1} \varphi_{0}+\lambda_{0} \varphi_{1}=0, \lambda=\left(\lambda_{0}, \lambda_{1}\right)$ étant les coordonnées homogènes de $t \in \mathbb{P}^{1}$. Par suite, d'après $F_{\lambda} \cdot \widetilde{\Sigma}=0$, on a $\Phi^{-1}(t) \cap \Sigma=\varnothing$ pour presque tout $t \in \mathbb{P}^{1}$. Compte tenu du fait que $\Sigma$ n'est pas exceptionnelle, $\Phi$ n'a pas de point d'indétermination et $\Sigma$ coïncide avec une composante connexe d'une fibre de $\Phi$. Par conséquent, la matrice $\left(\left(\Sigma_{i} \cdot \Sigma_{j}\right)\right)$ formée des nombres d'intersection des composantes irréductibles $\left\{\Sigma_{i}\right\}$ de $\Sigma$ est negative semi-définie et son déterminant $\left|\left(\Sigma_{i} \cdot \Sigma_{j}\right)\right|$ est nulle. On en déduit, par un calcule facile, que $\Sigma$ appartient au Tableau $3 \mathrm{du} \mathrm{n}^{\circ} 7$. Donc, les composantes connexes des fibres $\Phi^{-1}(t)$ voisines de $\Sigma$ sont elliptiques; c'est-à-dire, $S$ est une surface elliptique et $\Sigma$ est l'une de ses fibres (cf. K. Kodaira [11], II); on a donc, $\bar{\kappa}(V) \leqq 1$ (cf. [8], Theorem 4). $\quad$ C. Q.F.D.

De ces deux Propositions 7 et 8 , on obtient le

Théorème 7. Soit $S$ une surface complexe non-singulière et compacte, $C$ une courbe analytique (compacte) sur $S$. Supposons que $V=S-C$ est de type général (c'est-à-dire, $\bar{\kappa}(V)=2)$. Alors, toute application holomorphe 
$f: \Delta^{*} \rightarrow V$ du disque pointé $\Delta^{*}: 0<|z|<1$ dans $V$ telle que $f(0 ; V)$ soit vide s'étand en une application holomorphe $\bar{f} d u$ disque $\Delta:|z|<1$ dans $S$.

Corollaire (Un cas spécial du théorème de F. Sakai [24]). Soient $S$, $V$ les mêmes que dans le Théorème 7 ci-dessus. Alors, tout automorphisme analytique de $V$ est la restriction sur $V$ d'une transformation biméromorphe de $S$.

\section{Bibliographie}

[1] Brieskorn, E., Rationale Singularitäten komplexer Flächen. Inventioncs math., 4 (1968), 336-358.

[2] Brody, R., Compact manifolds and hyperbolicity, Trans. Amer. Math. Soc., 235 (1978), 213-219.

[3] Forster O. und Ramspott, K. J., Analytische Modulgarben und Endromisbündel, Inventiones math., 2 (1966), 145-170.

[4] Gunning R. C. and Narasimhan, R., Immersion of open Riemann surfaces, Math. Ann., 174 (1967), 103-108.

[5] Grauert, H., Über Modifikationen und exzeptionelle analytische Mengen, Math. Ann., 146 (1962), 331-368.

[6] Hirzebruch, F., Über vierdimensionale Riemannsche Flächen meherdeutiger analytischer Funktionen von zwei komplexen Veränderlichen, Math. Ann., 126 (1953), $1-22$.

[7] Hopf, H., Über komplex-analytische Mannigfaltigkeiten, Rend. Mat. e Appl. Serie $V, 10$ (1951), 161-182.

[8] Iitaka, S., On logarithmic Kodaira dimension of algebraic varieties, Complex Analysis and Algebraic Geometry, ed. by W. L. Baily and T. Shioda, Iwanami Shoten (1976), $175-189$.

[9] Kizuka, T., Analytic automorphisms and algebraic automorphism of $\boldsymbol{C}^{2}$, Tôhoku Math. J., 31 (1979), 553-565.

[10] Kobayashi, S., Hyperbolic manifolds and holomorphic mappings, Monographs in Pure and Applied Mathematics n ${ }^{\circ}$ 2, Marcel Dekker, New York, 1970.

[11] Kodaira, K., On compact complex analytic surfaces I, II, Ann. Math., 71 (1960), 111-152, 77 (1963), 563-626.

[12] - On the structure of compact complex analytic surfaces IV, Amer. J. Math., 90 (1968), 1048-1066.

[13] Kodaira K. and Spencer, D. C., A theorem of completeness of characteristic systems of complete continuous systems, Amer. J. Math., 81 (1959), 477-500.

[14] Nishino, T., Sur les ensembles pseudoconcaves, J. Math. Kyoto Univ., 1 (1962), 225-245.

[15] — Prolongements analytiques au sens de Riemann, Bull. Soc. Math. France, 107 (1979), 97-112.

[16] Noshiro, K., Cluster sets, Ergebnisse der Mathematik und ihren Grenzgebiete (neue Folge) 28, Springer-Verlag, Berlin-Göttingen-Heidelberg, 1960.

[17] Ohtsuka, M., On the behavior of an analytic function about an isolated boundary point, Nagoya Math. J., 4 (1952), 103-108. 
[18] - A theorem on cluster sets of an analytic mapping into a Riemann surface, Annales Acad. Sci. Fenicae Ser. A. I. Math., 2 (1976), 375-381.

[19] Oka, K., Note sur les familles de fonctions multiformes etc., J. Sci. Hiroshima Univ., (1934), 93-98.

[20] - Sur les fonctions analytiques de plusieures variables complexes IX, Domaines finis sans point critique intérieur, Japanese J. Math., 27 (1953), 97-155.

[21] Remmert R. und Stein, K., Über die wesentlichen Singularitäten analytischer Mengen, Math. Ann., 126 (1953), 263-306.

[22] Royden, H. L., Remarks on the Kobayashi metric, Lecture Notes in Math., 185, Springer-Verlag, 1971, 125-137.

[23] Šafarevič, R. I., Algebraic surfaces, Proceedings of the Steklov Inst. of Math., 75 1965. (Translation, Amer. Math. Soc. 1967.)

[24] Sakai, F., Kodaira dimensions of complements of divisors, le même libre que l'article de S. Iitaka [8], (1976), 239-257.

[25] Sario L. and Noshiro, K., Value distribution theory, Chap. VI, The Univ, series in Higher Math. D. van Nostrand Company, Inc., Princeton, New Jersey, etc., 1966.

[26] Siu, Y.-T., Every Stein subvariety admits a Stein neighborhood, Inventiones math., 38 (1976), 89-100.

[27] Suzuki, M., Sur les intégrales premières de certains feuilletages analytiques complexes, Séminaire François Norguet 1975-1976, Lecture Notes in Math., 670, Springer-Verlag, 1978, 53-88.

[28] Thullen, P., Über die wesentlichen Singularitäten analytischer Funktionen und Flächen in Raume von $n$ komplexen veränderlichen, Math. Ann., 111 (1934), 137-156. 
